Article type : Original

\title{
Methionine Adenosyltransferase $\alpha 1$ is targeted to the mitochondrial matrix and interacts with cytochrome P450 2E1 to lower its expression
}

Ben Murray ${ }^{1}$, Hui Peng ${ }^{1}$, Lucia Barbier-Torres ${ }^{1}$, Aaron Robinson ${ }^{2}$, Tony W. H. Li $^{1}$, Wei

Fan $^{1}$, Maria Lauda Tomasi ${ }^{1}$, Roberta A. Gottlieb ${ }^{3}$, Jenny Van Eyk ${ }^{2}$, Zhimin Lu$^{4}$, ML Martínez-Chantar ${ }^{5}$, Suthat Liangpunsakul ${ }^{6,7,8}$, Nicholas J Skill ${ }^{9}$, José M. Mato ${ }^{5}$, and Shelly C. $\mathrm{Lu}^{1}$

${ }^{1}$ Division of Digestive and Liver Diseases, Cedars-Sinai Medical Center, Los Angeles, CA 90048, USA; ${ }^{2}$ Advanced Clinical Biosystems Research Institute, Cedars-Sinai Medical Center, Los Angeles, CA 90048, USA; ${ }^{3}$ The Heart Institute, Cedars-Sinai Medical Center, Los Angeles, CA 90048, USA; ${ }^{4}$ Department of Neuro-Oncology, the University of Texas MD Anderson Cancer Center, Houston, Texas; ${ }^{5} \mathrm{CIC}$ bioGUNE, Centro de Investigación Biomédica en Red de Enfermedades Hepáticas y Digestivas (Ciberehd), Technology, Park of Bizkaia, 48160 Derio, Bizkaia, Spain; ${ }^{6}$ Division of Gastroenterology and Hepatology, Department of Medicine, Indiana University School of Medicine; ${ }^{7}$ Department of Biochemistry and Molecular Biology, Indiana University School of Medicine, Indianapolis, IN; ${ }^{8}$ Roudebush Veterans Administration Medical Center, Indianapolis, IN, ${ }^{9}$ Department of Surgery, Indiana University School of Medicine, Indianapolis, IN 46202

This is the author's manuscript of the article published in final edited form as:

Murray, B., Peng, H., Barbier-Torres, L., Robinson, A., Li, T. W. H., Fan, W., ... Lu, S. C. (n.d.). Methionine Adenosyltransferase $\alpha 1$ is targeted to the mitochondrial matrix and interacts with cytochrome P450 $2 \mathrm{E} 1$ to lower its expression. Hepatology, 0(ja). https://doi.org/10.1002/hep.30762 
Key words: Mitochondrial function, S-adenosylmethionine, methylation, alcoholic liver disease, proteasomal degradation

\section{FOOTNOTE PAGE}

Contact information: Shelly C. Lu, M.D., Division of Digestive and Liver Diseases, Department of Medicine, Cedars-Sinai Medical Center, Davis Building, Room \#2097, 8700 Beverly Blvd., Los Angeles, CA, 90048. Tel: (310) 423-5692, Fax: (310) 423-0653, e-mail: shelly.lu@cshs.org

Abbreviations used (alphabetical order): Ab, antibody; ALD, alcoholic liver disease; $\mathbf{C C l}_{4}$, carbon tetrachloride; ChIP, Chromatin Immunoprecipitation; CHX, cycloheximide; Co-IP, co-immunoprecipitation; CYP2E1, cytochrome P450 2E1; EM, electron microscopy; FBS, fetal bovine serum; GAPDH, glyceraldehyde 3-phosphate dehydrogenase; HCC, hepatocellular carcinoma; HSP, heat shock protein; IB, immunoblotting; Ig, Immunoglobulin G; IP, immunoprecipitation; KO, knockout; LC, liquid chromatography; MAT, methionine adenosyltransferase; mROS, mitochondrial ROS; MS, mass spectrometry; NAFLD, nonalcoholic fatty liver disease; OCR, oxygen consumption rate; PCR, polymerase chain reaction; PHB1, prohibitin 1; ROS, reactive oxygen species; SAMe, S-adenosylmethionine; SC, scramble siRNA; siRNA, small interfering RNA; TNF $\alpha$, tumor necrosis factor $\alpha$; WT, wild type; XIC, extracted ion intensities

Financial Support: This work was supported by NIH grants R01AA026759 (SC Lu, RA Gottlieb and J Van Eyk), R01DK107288 (SC Lu and J Van Eyk), and R01HL132075 (RA Gottlieb), Plan Nacional of I+D SAF 2014-52097R, MINECO-ISCiii PIE14/00031 (JM Mato and ML Martínez-Chantar) and CIBERehd-ISCiii (JM Mato and ML Martínez-Chantar) and the Erika Glazer Endowed Chair for Women's Health Heart and Barbara Streisand Women's 
Health Center (J Van Eyk). Part of the proteomics was performed by the Mass SpectrometryBased Proteomics Core of the USC Research Center for Liver Diseases (P30DK48522) and the Advanced Clinical Biosystems Research Institute at Cedars-Sinai Medical Center. Immunogold-EM was performed by S. Ryazantsev of the Electron Microscopy Services at EICN/CNSI University of California, Los Angeles. The funders had no role in study design, data collection and analysis, decision to publish, or preparation of the manuscript.

\section{ABSTRACT}

Methionine adenosyltransferase $\alpha 1$ (MAT $\alpha 1$, encoded by MAT1A) is responsible for hepatic biosynthesis of S-adenosylmethionine, the principal methyl donor. MAT $\alpha 1$ also act as a transcriptional co-factor by interacting and influencing the activity of several transcription factors. Matla knockout (KO) mice have increased levels of cytochrome P450 2E1 (CYP2E1), but the underlying mechanisms are unknown. The aims of the current study were to identify binding partners of MAT $\alpha 1$ and elucidate how MAT $\alpha 1$ regulates CYP2E1 expression. We identified binding partners of MAT $\alpha 1$ by co-immunoprecipitation (co-IP) and mass spectrometry. Interacting proteins were confirmed using co-IP using recombinant proteins, liver lysates and mitochondria. Alcoholic liver disease (ALD) samples were used to confirm relevance of our findings. We found MAT $\alpha 1$ negatively regulates CYP2E1 at mRNA and protein levels, with the latter being the dominant mechanism. MAT $\alpha 1$ interacts with many proteins, but with a predominance of mitochondrial proteins including CYP2E1. We found MAT $\alpha 1$ is present in the mitochondrial matrix of hepatocytes using immunogold electron microscopy. Matla $\mathrm{KO}$ hepatocytes had reduced mitochondrial membrane potential and higher mitochondrial reactive oxygen species, both of which were normalized when MATIA was overexpressed. In addition, KO hepatocytes were sensitized to ethanol and tumor necrosis factor $\alpha$-induced mitochondrial dysfunction. Interaction of MAT $\alpha 1$ with CYP2E1 was direct and this facilitated CYP2E1 methylation at R379 leading to its degradation via the proteasomal pathway. Matla KO livers have a reduced methylated/total CYP2E1 ratio. MAT $\alpha$ 1's influence on mitochondrial function is largely mediated by its effect on CYP2E1

This article is protected by copyright. All rights reserved. 
expression. Patients with ALD have reduced MATal levels and a decrease in methylated/total CYP2E1 ratio. Conclusion: Our findings highlight a critical role of MAT $\alpha 1$ in regulating mitochondrial function by suppressing CYP2E1 expression at multiple levels.

S-adenosylmethionine (SAMe) is the principal biological methyl donor of the cell that is synthesized by methionine adenosyltransferase (MAT), an enzyme encoded by the MAT gene, which is one of the 482 essential genes required for survival (1). Two genes in mammals (MAT1A and MAT2A) encode for the catalytic subunits MAT $\alpha 1$ and MAT $\alpha 2$. The former is expressed largely in normal liver and the latter is expressed in all extrahepatic tissues (1). Patients with chronic liver disease have reduced hepatic MAT activity, due to both a decrease in MATlA mRNA levels and inactivation of MAT $\alpha 1$ protein itself (1). MATIA expression is reduced in individuals with alcoholic hepatitis (2), non-alcoholic fatty liver disease (NAFLD) with more advanced fibrosis (3), in nearly $90 \%$ of cirrhotic patients (4) and in most hepatocellular carcinoma (HCC) (4-5). Matla knockout (KO) mice have chronic hepatic SAMe deficiency, spontaneously develop steatohepatitis and HCC (6-7). These mice are also sensitized to carbon tetrachloride $\left(\mathrm{CCl}_{4}\right)$-induced hepatotoxicity, due to higher cytochrome P450 2E1 (CYP2E1) expression (7) and display a decrease in mitochondrial membrane potential (8). However, the underlying mechanism for increased CYP2E1 expression is unknown and whether this contributes to a lower mitochondrial membrane potential in Matla $\mathrm{KO}$ mice is also unclear.

CYP2E1 has been implicated in numerous diseases and its expression is upregulated in alcoholic liver disease (ALD) (9-10) and NAFLD (11-12). CYP2E1 has been shown to be localized within several subcellular compartments including the ER, Golgi apparatus and within the mitochondrial matrix (13-17). It has been suggested that CYP2E1-mediated damage to mitochondrial membrane potential and function, through the production of reactive oxygen species (ROS), may be an early event in liver injury (9-10).

This article is protected by copyright. All rights reserved. 
We recently showed that MAT $\alpha 1$ interacts with proteins such as c-MYC and MAX to modulate E-box dependent gene expression $(18,19)$. The aims of this work were to further define what proteins could interact with MAT 1 and to explore how MAT $\alpha 1$ regulates CYP2E1 expression and mitochondrial function. Here, we revealed the novel finding that MAT $\alpha 1$ is localized to the mitochondrial matrix where it interacts with CYP2E1 to influence mitochondrial membrane potential and generation of ROS. Our results revealed a novel role of MATal in providing a mitochondrial source of SAMe in hepatocytes; and that it negatively regulates CYP2E1 protein stability via methylation at a critical arginine residue.

\section{EXPERIMENTAL PROCEDURES}

\section{Mat1a KO and wild type (WT) mice experiments}

Matla KO mice were previously described (6). Four-month old male KO and WT littermates were used for this study unless specified in the figure legend. Some mice were used for isolation of hepatocytes as described (20), whilst other mice livers were used for RNA, protein analysis and isolation of mitochondria as described in additional methods.

Female WT mice were treated with ethanol or pair-fed control diet following the protocol of the NIAAA model (21). Please see additional methods for details.

\section{Human samples}

Liver specimens from subjects with ALD $(\mathrm{N}=5)$ were obtained from the explant during liver transplantation. All had history of alcohol consumption averaging at least $80 \mathrm{~g} / \mathrm{d}$ for men or $50 \mathrm{~g} / \mathrm{d}$ for women, for at least 10 years (22). This criterion is based on epidemiological evidence of the alcohol consumption and cirrhosis relationship (23). We excluded patients with hepatitis $\mathrm{B}$ or $\mathrm{C}$, autoimmune liver disease, hemochromatosis, Wilson's disease, and HCC. Patients' clinical characteristics are shown in Supplementary Table 1. Liver samples from controls $(\mathrm{N}=4)$ were from subjects with no known history of excessive alcohol use or 
underlying chronic liver disease. They were obtained during abdominal surgeries for various causes.

\section{Mitochondrial membrane potential and ROS measurements}

Mitochondrial membrane potential was measured using the MitoProbe ${ }^{\mathrm{TM}} \mathrm{JC}-1$ (Molecular Probes, Eugene, OR) assay kit following the protocol provided. Mitochondrial ROS was measured using the MitoSOX ${ }^{\mathrm{TM}}$ Red kit (Molecular Probes, Eugene, OR) according to the manufacture's guide. Five $\mu \mathrm{M}$ antimycin A was added 10 minutes after (SML0737, Sigma, St Louis, MO) or $5 \mu \mathrm{M}$ of MitoTEMPO was added an hour prior to the start of the assay for positive and negative controls, respectively.

\section{Additional methods}

All other methods used are described in detail in the 'Supplemental Methods' section of the online supporting information.

\section{RESULTS}

\section{MATal interactome includes a predominance of mitochondrial proteins}

To define the MATal interactome, we performed immunoprecipitation (IP) with an antiMAT $\alpha 1$ antibody (Ab) using liver lysates from WT and Mat1a KO mice. Proteins were then separated on SDS-PAGE and identified using mass spectrometry (MS). Silver stain was used to highlight the differences between the WT and Matla KO mice samples (Supplemental Fig. 1A). Of the top 24 highest scoring proteins, 11 of them were mitochondrial (Supplemental Table 2). To further examine the MAT $\alpha 1$ interactome, GO and KEGG pathways enrichment analysis were performed using the STRING database (https://string-db.org/). Of the total 375 proteins, $40 \%$ were classified as mitochondrial with most of them associated to the mitochondrial matrix and inner mitochondrial membrane (Supplemental Fig. 1B-C). Additionally, the main mitochondrial metabolic pathways including glycolysis, the 
tricarboxylic cycle, and oxidative phosphorylation were all enriched (Supplemental Fig. 1D). CYP2E1, a protein that is elevated in the Matla $\mathrm{KO}$ mouse liver that can also target the mitochondria, was also identified (Supplemental Table 2).

\section{MATa1 is localized to the mitochondrial matrix of hepatocytes}

Since mitochondrial proteins account for such a high proportion of the MAT $\alpha 1$ interactome, we next examined whether MATal was localized within the mitochondria. Isolation of mitochondria from WT mice liver revealed the presence of MAT $\alpha 1$ within this organelle (Fig. 1A). To determine localization within the mitochondria, WT mice liver mitochondria were subjected to subfractionation with chemical agents to access the different compartments, followed by Western blotting with known marker proteins of each mitochondrial compartment, which revealed MAT $\alpha 1$ to be in the matrix as shown in the final column (Fig. 1B). To confirm the matrix location of MAT $\alpha 1$ and rule out the possibility of contamination from the other cellular compartments, immunogold electron microscopy (EM) was performed using WT mouse liver and the presence of MAT $\alpha 1$ can be seen as discrete concentric gold particles within the mitochondrial matrix and cytosol (Fig. 1C left panel) which are absent in the negative control (Fig. 1C right panel, Supplemental Fig. 2).

\section{MATa1 interacts with molecular chaperones and its mitochondrial targeting requires HSP70A8}

MAT $\alpha 1$ 's presence in the mitochondrial matrix of hepatocytes prompted us to question how this protein is targeted to this organelle. Since MAT $\alpha 1$ contains no mitochondrial targeting sequence to aid localization to the mitochondria as determined by multiple organelle localization prediction servers (TargetP, Multiloc and MitoFates), other known mechanisms of protein trafficking to the mitochondria were studied, namely the use of heat shock proteins (HSP) (24). HSP70A8 and HSP90 3 , HSPs known to be involved in protein trafficking, were identified to interact with MAT $\alpha 1$ (Supplemental Table 2). IPs using WT mouse liver were carried out and showed that MAT $\alpha 1$ interacts with the cytosolic molecular chaperones HSP70A8 and HSP90 $\beta$ (Fig 2A-B). When HSPA8 was knocked down in HepG2 cells,

This article is protected by copyright. All rights reserved. 
MAT $\alpha 1$ levels decreased in the mitochondrial fraction by $45 \%$ suggesting that HSP70A8 plays an important role in MAT $\alpha 1$ localization to the mitochondria (Fig 2C-D, and second siRNA shown in Supplemental Fig. 3A). In contrast, when HSP90AB1 was knocked down, mitochondrial MAT $\alpha 1$ also fell by $32 \%$ but this was due to a drop by the same amount of cytosolic MAT $\alpha 1$ (Fig. 2E-F, and second siRNA shown in Supplemental Fig. 3B).

\section{MATa1 affects mitochondrial function, mitochondrial ROS and mitochondrial membrane potential}

It was previously reported that Matla $\mathrm{KO}$ mice had reduced mitochondrial membrane potential (8), so functional experiments were explored to evaluate the effect of MAT $\alpha 1$ on mitochondrial function. Consistent with previously published data (8), we found the hepatocytes from the Matla $\mathrm{KO}$ mouse showed a $40 \%$ reduction in mitochondrial membrane potential as compared to WT hepatocytes, which could be restored upon the overexpression of MATIA (Fig. 3A). Levels of mitochondrial ROS (mROS) were elevated in Matla KO hepatocytes by $30 \%$ and were normalized to WT level after MAT1A overexpression (Fig. 3A). To rule out the possibility of spurious signals in the mROS experiments, we included either antimycin $\mathrm{A}$, an inhibitor to the mitochondrial respiration complex III, or the superoxide scavenger mitoTEMPO as positive and negative controls, respectively (Supplemental Fig. 3C-E). Using WT hepatocytes, overexpressing MATlA increased the mitochondrial membrane potential (25\%) whilst silencing caused the opposite effect (Fig. 3B, and second siRNA shown in Supplemental Fig. 4A). mROS levels fell in WT hepatocytes upon the overexpression of MATIA (25\%), and levels increased by $30 \%$ when MATIA was silenced (Fig. 3B, and second siRNA shown in Supplemental Fig. 4A). Silencing MAT1A in HepG2 cells had the same effect as in WT hepatocytes, lower membrane potential (20\%) and higher mROS levels (35\%) as compared to the scramble control (Fig. 3C, and second siRNA shown in Supplemental Fig. 4B).

This article is protected by copyright. All rights reserved. 
To examine whether Matla KO hepatocytes are sensitized to stress, mitochondrial membrane potential and mROS were measured after treatment with either tumor necrosis factor $\alpha$ $(\mathrm{TNF} \alpha)$ or ethanol. TNF $\alpha$ had no significant effect on WT hepatocytes but it caused a $17 \%$ decrease in membrane potential and a $40 \%$ increase in mROS levels in Matla $\mathrm{KO}$ hepatocytes (Supplemental Fig. 4C). Interestingly, ethanol addition resulted in a 50\% increase in membrane potential of WT hepatocytes, but lowered the membrane potential of Matla KO hepatocytes by 22\% (Supplemental Fig. 4D). Ethanol induced mROS in WT hepatocytes by $50 \%$ but caused nearly a 2-fold increase in Matla $\mathrm{KO}$ hepatocytes (Supplemental Fig. 4D).

To further study the effects of MAT $\alpha 1$ on mitochondrial function we performed respirometry experiments. The oxygen consumption rate (OCR) was $30 \%$ lower in isolated liver mitochondria from Matla KO mice as compared to WT (Fig. 3D). Silencing of MAT1A in HepG2 cells significantly decreased basal respiration, ATP production, the maximum respiratory capacity, and the reserve capacity (Fig 3E, and second siRNA shown in Supplemental Fig. 4E).

\section{MATa1 and CYP2E1 interact with each other directly}

CYP2E1, a protein known to target to mitochondria and one that generates ROS, was identified by MS as a potential binding partner of MAT $\alpha 1$ (Supplemental Table 2). To confirm this interaction, we performed pulldown assays with purified recombinant proteins and a series of IP experiments. Direct interaction between CYP2E1 and MAT $\alpha 1$ was demonstrated using recombinant proteins (Fig. 4A-B), whilst endogenous interaction was demonstrated by co-IP in both mouse liver lysates (Fig. 4C) and in isolated mouse liver mitochondria (Fig. 4D).

This article is protected by copyright. All rights reserved. 


\section{MATa1 negatively regulates CYP2E1 expression}

We next examined how MAT $\alpha 1$ influences CYP2E1 expression and the functional outcome of their interaction. Levels of Cyp2el mRNA were increased by $50 \%$ in Mat1a $\mathrm{KO}$ mice livers compared to the WT control (Fig 5A), whilst CYP2E1 protein levels in the Matla KO livers were 4.6-fold of the WT controls, suggesting the predominant mechanism for increased CYP2E1 expression lies at the protein level (Fig. 5B). Isolated mitochondria from WT and Matla KO mice livers showed a 54\% increase in mitochondrial CYP2E1 level in the Matla KO (Fig. 5C). This increase was also confirmed using MS (Fig 5D). Since the dominant mechanism of MATa1-mediated suppression of CYP2E1 lies at the protein level we investigated how this occurs and the functional consequences.

\section{MAT1A knockdown-mediated mitochondrial dysfunction is CYP2E1 dependent}

CYP2E1 is known to influence mitochondrial function (10), which prompted us to examine whether MAT1A-mediated changes in CYP2E1 expression could play an important role in mitochondrial function. Knockdown of MATIA in WT mouse hepatocyte cells doubled CYP2E1 protein abundance; whilst knockdown of CYP2E1 did not change the level of MAT $\alpha 1$ expression, it prevented MAT1A knockdown-mediated CYP2E1 induction (Fig. 5E, and second siRNA shown in Supplemental Fig. 5A). Knockdown of MAT1A decreased mitochondrial membrane potential by $25 \%$ whilst mROS levels increased by $25 \%$. Knockdown of $C Y P 2 E 1$ resulted in a $30 \%$ increase in mitochondrial membrane potential and a 30\% decrease in mROS. Combined knockdown of MATIA and CYP2E1 caused no change in membrane potential but the increase in mROS after MAT1A knockdown was blocked (Fig. 5F, and second siRNA shown in Supplemental Fig. 5B).

\section{CYP2E1 is methylated and methylated-CYP2E1 (me-CYP2E1) level is reduced in Mat1a}

\section{KO liver mitochondria}

Interaction of MAT $\alpha 1$ and CYP2E1 raised the possibility that CYP2E1 might be methylated and this could regulate its protein stability. Co-IP using both WT and Matla KO mice liver mitochondria with anti-mono/di-methyl arginine $\mathrm{Ab}$ followed by immunoblotting (IB) for 
CYP2E1 revealed CYP2E1 to be methylated and methylated CYP2E1 (me-CYP2E1) levels were higher in the WT mitochondria compared to the Mat1a KO, while total CYP2E1 protein levels were higher in the Mat1a KO, resulting in an $70 \%$ decrease in me-CYP2E1/total CYP2E1 ratio (Fig. 6A). The decrease in methylated CYP2E1 in Matla KO mouse liver mitochondria was also confirmed using MS (Fig. 6B-C).

\section{MATa1-mediated down-regulation of CYP2E1 protein level requires $R 379$ methylation}

To identify the possible site of methylation on CYP2E1, methylation prediction servers were run using the human CYP2E1 protein sequence which led to the identification of two arginine residues, R100 and R379 (Supplemental Table 3). Mutation of these residues to asparagine, both single and double mutations, and overexpression in HepG2 cells showed that they were stably expressed to a level comparable to WT CYP2E1 (Fig 6D). Interestingly, when MAT1A was also overexpressed a clear reduction in WT and R100N CYP2E1 was observed (50\%), but this reduction did not occur when $\mathrm{R} 379 \mathrm{~N}$ or the double mutant CYP2E1 was overexpressed, suggesting that R379 is critical for MAT $\alpha 1$-dependent CYP2E1 degradation (Fig. 6D).

\section{MATa1 reduces the half-life of CYP2E1 and aids its degradation through the proteasomal pathway}

To examine the effect that methylation had on the stability of CYP2E1, CYP2E1 was overexpressed alone or with MAT1A prior to using cycloheximide (CHX) to stop de novo protein synthesis in HepG2 cells. Overexpression of MAT1A significantly reduced the $t_{1 / 2}$ of co-expressed CYP2E1 by almost 40\% (Fig. 7A-B). To see whether the effect of MAT1A overexpression is mediated by enhanced proteasomal degradation of CYP2E1, HepG2 cells were transfected with CYP2E1-DDK, with or without MATIA overexpression vectors and treated with the proteasomal inhibitor MG132. Figure 7C shows that MG132 completely abolished MAT1A overexpression-mediated down-regulation of CYP2E1 protein level. We further examined the effects of MG132 by performing a time course over six hours. CYP2E1 protein levels remained relatively stable over six hours, but upon addition of MG132 
CYP2E1 levels nearly doubled over the same time course (Supplemental Fig. 6A). When MAT1A is co-overexpressed there is a slight decrease in the levels of CYP2E1, which was blocked upon MG132 treatment (Supplemental Fig. 6B).

As MAT1A overexpression-mediated down-regulation of CYP2E1 protein level seemed to occur through the proteasomal degradation pathway we examined whether MAT $\alpha 1$ was also localized in the microsome. Upon isolation of microsomes from mouse liver, the presence MAT $\alpha 1$ could be seen via Western blot, along with calnexin, a marker of the endoplasmic reticulum, and absence of tubulin, a marker of the cytosol, to show the purity of the isolation (Fig 7D). Further IP studies revealed that MAT $\alpha 1$ and CYP2E1 also interact in this subcellular compartment (Fig 7E-F).

It has been reported that levels of CYP2E1 fall during hepatocyte dedifferentiation (25), so we compared CYP2E1 protein expression in WT and Matla KO hepatocytes cultured for 48 hours. Consistent with previous reports (25), levels of CYP2E1 fell in WT hepatocytes by $50 \%$ over 48 hours. CYP2E1 levels also fell in Mat1a KO hepatocytes but by 48 hours its level was much higher than that of WT hepatocytes (Supplemental Fig. 6C).

\section{Levels of CYP2E1 and me-CYP2E1 are increased in human ALD and a mouse model of} chronic and binge ethanol feeding

To extend our observations to pathophysiological state in humans, we determined the mitochondrial presence of MAT $\alpha 1$ and its interaction with CYP2E1, levels of MAT $\alpha 1$ protein as well as methylated CYP2E1 levels in the liver tissues of subjects with ALD, a known liver condition with increased protein expression of CYP2E1. Normal human liver showed the presence of MATal within the mitochondria (Fig. 8A). The endogenous interaction of MAT $\alpha 1$ and CYP2E1 was confirmed by performing co-IP (Fig. 8B). Consistent with lower MAT1A mRNA levels in ALD (2), MAT 1 1 protein levels are reduced in ALD as compared to normal controls (Fig. 8C). CYP2E1 is methylated in human liver samples and 
those from ALD patients have higher total CYP2E1 level but a lower me-CYP2E1 to total CYP2E1 ratio as compared to normal controls (Fig. 8D), which was similar to what's observed in the Mat1a KO animal model (Fig 6A). Furthermore, similar to human ALD, samples from the NIAAA mouse model, a model of chronic and binge ethanol feeding (21), showed that the me-CYP2E1 to total CYP2E1 ratio was lower as compared to pair-fed controls (Fig. 8E).

\section{DISCUSSION}

Up until recently the general understanding has been that MATlA-encoded isoenzymes are cytosolic and provide SAMe biosynthesis in normal liver. It is well accepted that MAT1A expression and SAMe levels fall in chronic liver disease and HCC (1). Mice lacking Matla have higher total lipid peroxides in serum, altered mitochondrial membrane potential, are sensitized to $\mathrm{CCl}_{4}$-induced hepatotoxicity due to higher CYP2E1 expression, and spontaneously develop steatohepatitis and HCC (6-7). Much of the phenotype of Matla KO mice was thought to be consequences of cytosolic SAMe deficiency, although how SAMe deficiency resulted in increased CYP2E1 expression was unclear. More recently others and we have shown MAT $\alpha 1$ can target the nuclear compartment where it can regulate gene expression by epigenetic mechanisms (26-28). In addition, we recently showed MAT $\alpha 1$ interacts directly with MAX and c-MYC and negatively regulate E-box-driven reporter activity (19). This prompted us to investigate the MAT $\alpha 1$ interactome, which revealed the unexpected high number of mitochondrial proteins, including CYP2E1 (Supplemental Table 2). In the course of these investigations we unveiled two main novel findings. First is that MAT $\alpha 1$ is targeted to the mitochondria where it plays an important role in regulating mitochondrial function, and second is that MAT $\alpha 1$ 's importance in mitochondrial function is related to its ability to negatively regulate CYP2E1 expression.

This article is protected by copyright. All rights reserved. 
It has been known for a long time that Matla $\mathrm{KO}$ mice have lower mitochondrial membrane potential, which was thought to be due to reduced prohibitin 1 (PHB1) expression (8). PHB1 is an important mitochondrial chaperone protein whose stability is regulated by the SAMe level (8). However, when we noticed that $40 \%$ of all the interacting proteins and $46 \%$ of the top MATal interacting proteins were mitochondrial, we examined more closely whether MAT $\alpha 1$ could also target the mitochondria (Supplemental Fig. 1, Supplemental Table 2). Using a variety of tools from co-IP, mitochondrial subfractionation to finally immunogold EM, we demonstrated the unequivocal presence of MAT $\alpha 1$ in the mitochondrial matrix (Fig. 1, Supplemental Fig. 2). Previous investigations highlighted the importance of SAMe within the mitochondria, which led to the identification of the SAMe channel transporter, SLC25A26, that was believed to be the only mechanism to allow SAMe entry into the mitochondrial matrix in cells (29). Members of the SLC25 family encode for mitochondrial carriers that are membrane embedded proteins, which translocate solutes across the inner mitochondrial membrane (29-30). The importance of SAMe within the mitochondria can be seen in patients that have mutations resulting in non-functional SLC25A26 proteins. Three cases of SLC25A26 mutations have been reported where a lack of a functional channel caused lactic acidosis, cystic necrosis of the germinal matrix, and cardiopulmonary failure (31). Interestingly, there was no mention of any liver abnormality. Although hepatocytes also express this channel (data not shown), presence of MAT $\alpha 1$ in the mitochondrial matrix suggest it can also provide a local source of SAMe, which may be necessary as SAMe has a half-life of only about $5 \mathrm{~min}$ (32) and is competed for by hundreds of enzymes (33). The fact that liver is where $85 \%$ of all transmethylation reactions take place (1) supports the notion that having MAT 1 near important substrates in the mitochondria facilitates their regulation by methylation.

Targeting of MAT $\alpha 1$ to the mitochondrial matrix essentially involves two stages: i) targeting to, and recognition by the mitochondria and ii) translocation through the outer and inner membrane by import machinery. MAT $\alpha 1$ does not contain the classical amino-terminal cleavable presequence for mitochondrial targeting as determined by TargetP, Multiloc and

This article is protected by copyright. All rights reserved. 
MitoFates, or an internal mitochondrial intermembrane space signal that would directly target it to the mitochondria (34). One well-established method of mitochondrial protein recruitment is through HSPs, which play a major role in trafficking proteins to and across the mitochondria membranes (24). From MS results, multiple HSPs were found to potentially interact with MAT $\alpha 1$, including the constitutively expressed cytosolic chaperones, HSP70A8 and HSP90ß, as well as several mitochondrial matrix HSPs, including HSP60, HSP70A9 and TRAP-1 (Supplemental Table 2). Co-IP experiments using mouse liver lysate showed that MAT $\alpha 1$ interacted with the cytosolic HSP proteins HSP70A8 and HSP90 $\beta$, suggesting that they may facilitate successful transport of MAT $\alpha 1$ to the mitochondria (Fig. 2A-B). Our finding that knockdown of $H S P A 8$ reduced the amount of MAT $\alpha 1$ in the mitochondria by 45\% (Fig. 2C-D, Supplemental Fig. 3A) is consistent with the notion that HSP70A8 plays an important role in MAT $\alpha 1$ mitochondrial targeting. Interestingly silencing of HSP90AB1 reduced the cytosolic and mitochondrial MAT $\alpha 1$ fraction by around $40 \%$ suggesting that this chaperone may play a role in the maturation of MAT $\alpha 1$ rather than aiding its translocation to the mitochondria (Fig. 2E-F, Supplemental Fig. 3B). Further studies are needed to elucidate the precise mechanism of entry into the mitochondrial matrix.

Even though Matla $\mathrm{KO}$ mice livers are known to have lower mitochondrial membrane potential (8), mitochondrial function had never been directly assessed. Utilizing the mitochondrial Seahorse analyzer with both a cellular system and isolated mitochondria, we examined whether MAT $\alpha 1$ modulates mitochondrial respiration and energy production in the liver. For that, we isolated liver mitochondria from WT and Matla KO mice and measured real-time oxygen consumption as a readout of oxidative phosphorylation. In addition, to be able to determine key parameters of mitochondrial function we silenced MAT1A in HepG2 cells. As expected, our studies show that MAT $\alpha 1$ positively regulates mitochondrial function in the liver both in vitro and in vivo. Its absence significantly decreased ADP-stimulated respiration in isolated liver mitochondria (Fig. 3D) and basal respiration and ATP-linked respiration in HepG2 cells (Fig. 3E, Supplemental Fig. 4E). The maximal respiratory capacity, which shows the maximum rate of respiration that the cell can achieve and mimics

This article is protected by copyright. All rights reserved. 
the physiological energy demand, and also the reserve capacity, which is an estimate of the potential bioenergetic reserve the cell can call upon in times of stress, were significantly reduced upon MATIA silencing. We also found that Matla $\mathrm{KO}$ hepatocytes were much more susceptible to the effects of TNF $\alpha$ and ethanol on mitochondrial function. TNF $\alpha$ had little effect on WT hepatocytes but raised mROS and lowered mitochondrial membrane potential in Matla KO hepatocytes (Supplemental Fig 4C). Similarly, ethanol raised mROS levels much more in $\mathrm{KO}$ hepatocytes. Intriguingly, ethanol treatment increased membrane potential in WT hepatocytes but lowered it in KO hepatocytes (Supplemental Fig. 4C). Our results are consistent with a previous report that showed ethanol hyperpolarized mitochondrial membrane potential in mouse myocardial cells (35). Taken together, these results support a major role of MAT $\alpha 1$ in mitochondrial function.

Amongst the MAT $\alpha 1$ interacting proteins we focused on CYP2E1 because our earlier study showed Matla $\mathrm{KO}$ mice are sensitized to $\mathrm{CCl}_{4}$-induced liver injury and this was due to increased CYP2E1 expression and activity (7). CYP2E1 is an important target as its induction is implicated in a variety of human diseases, including diabetes, ALD and NAFLD (36). A marked increase in mitochondrial CYP2E1 content is associated with mitochondrial oxidative stress, suggesting a direct role of CYP2E1 in ROS production $(17,37)$. Our study found that MAT $\alpha 1$ regulates CYP2E1 expression negatively by two different mechanisms. One is at the mRNA level as Cyp2el mRNA level increased by $50 \%$ in Matla $\mathrm{KO}$ mice (Fig. 5A) and the second is at the protein level, as Mat1a KO mice had a 4.6-fold increase in CYP2E1 in total protein level (Fig. 5B). CYP2E1 induction in many instances (such as by alcohol) also occurs largely at the post-translational level by regulating its proteasomal degradation (10). This prompted us to devote our efforts to elucidate how MAT $\alpha 1$ regulates CYP2E1 at the protein level and examine the functional consequences.

This article is protected by copyright. All rights reserved. 
We found that recombinant MAT $\alpha 1$ and CYP2E1 can directly interact in vitro, and endogenous proteins interact in total, mitochondrial, and microsomal lysates (Fig. 4, Fig. 7EF). Since we speculated that mitochondrial MAT $\alpha 1$ may be providing a local source of SAMe to methylate its targets, we next examined whether CYP2E1 might be methylated. Indeed, we found lower methylated CYP2E1 but higher total CYP2E1 in the KO mitochondria (Fig. 6A), suggesting methylation of CYP2E1 may destabilize the protein. This was also true in human $\mathrm{ALD}$, a disease where MAT $\alpha 1$ is decreased, CYP2E1 is increased and total liver lysate showed a reduction in me-CYP2E1 (Fig. 8C-D). A similar decrease in me-CYP2E1 to total CYP2E1 ratio also occurred in the NIAAA mouse model (Fig. 8E). Methylation prediction servers highlighted several arginine residues as possible candidates but did not predict any lysine residues (Supplemental Table 3). Analysis of the human CYP2E1 crystal structure (PDB:3T3Z) showed that R379 would be the most likely target of methylation as its side chain was surface accessible unlike R100 whose side chain was involved in forming hydrogen bonds with the protoporphyrin IX moiety of CYP2E1 (Supplemental Fig. 7A-C). Mutagenesis studies confirmed that R379 of CYP2E1 is the critical residue that is required for MAT $\alpha 1$ overexpression to destabilize CYP2E1 (Fig 6D). To the best of our knowledge CYP2E1 has not been shown to be methylated. Although our current study is mainly focused on the mitochondria, it is highly likely that methylation of CYP2E1 is also occurring in the other cellular compartments such as the ER and may similarly affect its stability, as we also found MAT 1 to be present in the microsomal fraction where it interacts with CYP2E1 (Fig 7D-F).

We also examined the effect MAT $\alpha 1$ had on the degradation of CYP2E1. Overexpression of MAT $\alpha 1$ reduced the amount of CYP2E1 protein, but this effect was blocked upon the addition of MG132, a proteasomal inhibitor (Fig. 7C), supporting our notion that methylation of CYP2E1 at R379 enhances CYP2E1 degradation through the proteasomal system. To this, upon blocking of de novo protein synthesis by $\mathrm{CHX}$, addition of MAT1A reduced the $t_{1 / 2}$ of CYP2E1 from 5 to 3 hours (Fig. 7A-B), further providing evidence that methylation of CYP2E1 leads to its decreased protein stability. The rapid phase degradation of CYP2E1 has

This article is protected by copyright. All rights reserved. 
been reported to have a $t_{1 / 2}$ between 3-6 hours in rat hepatoma cells (38-39) and between 2.56 hours in HepG2 cells expressing human CYP2E1 (40), which is consistent with our findings. Although there are proteases within the mitochondria there is no proteasomal system, so how mitochondrial methylated CYP2E1 could be degraded is not clear. One possible explanation is that upon methylation, CYP2E1 is transported out of the mitochondria for degradation via the proteasomal degradation system. There is evidence of proteins being transported out of the mitochondria as a recent report by Sutendra et al (41) described the accumulation of pyruvate dehydrogenase in the nucleus in the S-phase of the cell cycle where it participated in histone acetylation. It was devoid of presequences, suggesting that the proteins interacted with mitochondrial processing peptidase prior to nuclear translocation. Lavie et al (42) recently published that the inner mitochondrial matrix protein SDHA was found ubiquitinated within the mitochondrial but did not identify if this modification occurred before protein entry into the mitochondria. Although the mitochondrial matrix is not accessible to the cytosolic proteasome, the outer mitochondrial membrane is. Ubiquitination of key mitochondrial outer membrane proteins can alter mitochondrial dynamics through fusion (mitofusins) and fission (DRP1 and FIS1) of mitochondria (43). The ubiquitination proteasome-mediated degradation of the mitofusin proteins MFN1 and MFN2 in damaged mitochondria is necessary in order to prevent fusion with healthy mitochondria and favor fragmentation instead (43). Whether loss of MAT1A leads to a difference in the mitochondrial ubiquitination pattern is an intriguing topic for future investigation.

Since an increase in mitochondrial CYP2E1 can cause mitochondrial dysfunction (10), we examined what role increased CYP2E1 expression played in the MAT1A knockdownmediated mitochondrial dysfunction. We found MAT1A knockdown mediated fall in mitochondrial membrane potential and increase in mROS required CYP2E1 induction (Fig. 5E-F), which support an important role for CYP2E1. However, we cannot exclude participation of other mitochondrial proteins that interact with MAT $\alpha 1$, such as ATP synthase (Supplemental Table 2). Future studies will examine whether these proteins are methylated and whether methylation alters their functionality.

This article is protected by copyright. All rights reserved. 
In summary, our current study showed MAT $\alpha 1$ is targeted to the mitochondrial matrix in hepatocytes to provide an immediate source of SAMe to important substrates that are regulated by methylation. MAT $\alpha 1$ negatively regulates CYP2E1 expression mainly at the protein levels. Our data support CYP2E1 methylation at R379 enhances its proteasomal degradation. These findings may help explain the consequences of MAT1A down-regulation that occur in many chronic human diseases including ALD where there is a decrease in MAT 1 1, me-CYP2E1 and an increase in total CYP2E1 levels.

\section{REFERENCES:}

1. Lu SC, Mato JM. S-adenosylmethionine in liver health, injury, and cancer. Physiol Rev 2012;92(4):1515-1542.

2. Lee TD, Sadda MR, Mendler MH, Bottiglieri T, Kanel G, Mato JM, et al. Abnormal hepatic methionine and glutathione metabolism in patients with alcoholic hepatitis. Alcohol Clin Exp Res 2004;28(1):173-181.

3. Murphy SK, Yang H, Moylan CA, Pang H, Dellinger A, Abdelmalek MF, et al. Relationship between methylome and transcriptome in patients with nonalcoholic fatty liver disease. Gastroenterology 2013;145(5):1076-1087.

4. Torres L, Avila MA, Carretero MV, Latasa MU, Caballería J, López-Rodas G, et al. Liver-specific methionine adenosyltransferase MAT1A gene expression is associated with a specific pattern of promoter methylation and histone acetylation: implications for MAT1A silencing during transformation. FASEB J 2000;14(1):95-102.

5. Cai J, Sun WM, Hwang JJ, Stain SC, Lu SC. Changes in S-adenosylmethionine synthetase in human liver cancer: molecular characterization and significance. Hepatology 1996;24(5):1090-1097.

6. Lu SC, Alvarez L, Huang ZZ, Chen L, An W, Corrales FJ, et al. Methionine adenosyltransferase 1A knockout mice are predisposed to liver injury and exhibit 
increased expression of genes involved in proliferation. Proc. Natl. Acad. Sci. U.S.A. 2001;98(10):5560-5565.

7. Martínez-Chantar ML, Corrales FJ, Martínez-Cruz LA, García-Trevijano ER, Huang ZZ, Chen L, et al. Spontaneous oxidative stress and liver tumors in mice lacking methionine adenosyltransferase 1A. FASEB J 2002;16(10):1292-1294.

8. Santamaría E, Matías A, Avila M, Latasa U, Rubio A, Martín-Duce A, et al. Functional proteomics of nonalcoholic steatohepatitis: Mitochondrial proteins as targets of Sadenosylmethionine. Proc. Natl. Acad. Sci. U.S.A. 2003;100(6):3065-3070.

9. Caro AA, Cederbaum AI. Oxidative stress, toxicology, and pharmacology of CYP2E1. Annu. Rev. Pharmacol. Toxicol 2004;44:27-42.

10. Lu Y, Cederbaum AI. CYP2E1 and oxidative liver injury by alcohol. Free Radic. Biol. Med 2008;44(5):723-738.

11. Aubert J, Begriche K, Knockaert L, Robin MA, Fromenty B. Increased expression of cytochrome P450 2E1 in nonalcoholic fatty liver disease: Mechanisms and pathophysiological role. Clin. Res. Hepatol. Gastroenterol 2011;35(10):630-637.

12. Abdelmegeed MA, Banerjee A, Yoo SH, Jang S, Gonzalez FJ, Song BJ. Critical role of cytochrome P450 2E1 (CYP2E1) in the development of high fat-induced non-alcoholic steatohepatitis. J. Hepatol. 2012;57(4): 860-866.

13. Neve EPA, Eliasson E, Pronzato MA, Albano E, Marinari U, Ingelman-Sundberg M. Enzyme-specific transport of rat liver cytochrome P450 to the golgi apparatus. Arch. Biochem. Biophys 1996;333(2):459-465.

14. Neve EP, Ingelman-Sundberg M. Molecular basis for the transport of cytochrome P450 2E1 to the plasma membrane. J. Biol. Chem 2000;275(22):17130-17135.

15. Neve EP, Ingelman-Sundberg M. Identification and characterization of a mitochondrial targeting signal in rat cytochrome P450 2E1 (CYP2E1). J. Biol. Chem 2001;276(14):11317-11322.

16. Robin MA, Anandatheerthavarada HK, Biswas G, Sepuri NB, Gordon DM, Pain D, et al. Bimodal targeting of microsomal CYP2E1 to mitochondria through activation of an N-

This article is protected by copyright. All rights reserved. 
terminal chimeric signal by cAMP-mediated phosphorylation. J. Biol. Chem 2002;277(43):40583-40593.

17. Raza H, Prabu SK, Robin MA, Avadhani NG. Elevated mitochondrial cytochrome P450 2E1 and glutathione S-transferase A4-4 in streptozotocin-induced diabetic rats. Diabetes 2003;53(1):185-194.

18. Yang H, Liu T,Wang J, Li TW, Fan W, Peng H, et al. Deregulated methionine adenosyltransferase $\alpha 1$, c-Myc, and Maf proteins together promote cholangiocarcinoma growth in mice and humans. Hepatology 2016;64(2):439-455.

19. Fan W, Yang H, Liu T, Wang J, Li TWH, Mavila N, et al. Prohibitin 1 Suppresses Liver Cancers Tumorigenesis in Mice and Humans. Hepatology 2017;65(4):1249-1266.

20. Kreamer BL, Staecker JL, Sawada N, Sattler GL, Hsia MT, Pitot HC. Use of a lowspeed, ISO-density percoll centrifugation method to increase the viability of isolated rat hepatocyte preparations. In Vitr. Cell. Dev. Biol 1986;22(4): 201-211.

21. Bertola A, Matthews S, Ki SH, Wang H, Gao B. Mouse model of chronic and binge ethanol feeding (the NIAAA model). Nature Protocols 2013;8(3): 627-637.

22. Whitfield JB, Rahman K, Haber PS, Day CP, Masson S, Daly AK, et al. Brief report: genetics of alcoholic cirrhosis—genomALC multinational study. Alcohol. Clin. Exp. Res 2015;39(5):836-842.

23. Cutright P, Fernquist RM. Predictors of per capita alcohol consumption and genderspecific liver cirrhosis mortality rates: thirteen European countries, Circa 1970-1984 and 1995-2007. OMEGA. J. Death Dying 2010;62(3):269-283.

24. Stojanoversus ki D, Bohnert M, Pfanner N, van der Laan M. Mechanisms of protein sorting in mitochondria. Cold Spring Harb. Perspect. Biol 2012;4(10):a011320.

25. Yang JW, Shin JS, Lee JJ, Chang HI, Kim CW. In vitro model using mouse hepatocytes for study of alcohol stress. Biosci Biotechnol Biochem 2001;65(7):1528-33.

26. Reytor E, Pérez-Miguelsanz J, Alvarez L, Pérez-Sala D, Pajares MA. Conformational signals in the C-terminal domain of methionine adenosyltransferase I/III determine its nucleocytoplasmic distribution. FASEB J 2009;23(10):3347-3360.

This article is protected by copyright. All rights reserved. 
27. Yang H, Cho ME, Li TW, Peng H, Ko KS, Mato JM, et al. MicroRNAs regulate methionine adenosyltransferase 1A expression in hepatocellular carcinoma. J. Clin. Invest 2013;123(1):285-298.

28. Pérez C, Pérez-Zúñiga FJ, Garrido F, Reytor E, Portillo F, Pajares MA. The oncogene PDRG1 is an interaction target of methionine adenosyltransferases. PLoS One 2016;11(8):e0161672.

29. Agrimi G, Di Noia MA, Marobbio CMT, Fiermonte G, Lasorsa FM, Palmieri F. Identification of the human mitochondrial S-adenosylmethionine transporter: bacterial expression, reconstitution, functional characterization and tissue distribution. Biochem. $\mathrm{J}$ 2004;379(Pt.1):183-190.

30. Palmieri F. The mitochondrial transporter family SLC25: Identification, properties and physiopathology. Mol. Aspects Med 2013;34(2-3):465-484.

31. Kishita Y, Pajak A, Bolar NA, Marobbio CM, Maffezzini C, Miniero DV, et al. Intramitochondrial methylation deficiency due to mutations in SLC25A26. Am. J. Hum. Genet 2015;97(5):761-768.

32. Mudd SH, Poole JR. Labile methyl balances for normal humans on various dietary regimens. Metabolism 1975;24(6):721-735.

33. Petrossian TC, Clarke SG. Multiple motif scanning to identify methyltransferases from the yeast proteome. Mol. Cell. Proteomics 2009;8(7):1516-1526.

34. Chacinska A, Koehler CM, Milenkovic D, Lithgow T, Pfanner N. Importing mitochondrial proteins: machineries and mechanisms. Cell 2009;138(4):628-644.

35. Mashimo K, Ohno Y. Ethanol hyperpolarizes mitochondrial membrane potential and increases mitochondrial fraction in cultured mouse myocardial cells. Arch Toxicol 2006;80(7):421-8.

36. Leung TM, Nieto N. CYP2E1 and oxidant stress in alcoholic and non-alcoholic fatty liver disease. J. Hepatol 2013;58(2):395-398.

37. Qi X, Miao L, Cai Y, Gong L, Ren J. ROS generated by CYP450, especially CYP2E1, mediate mitochondrial dysfunction induced by tetrandrine in rat hepatocytes. Acta Pharmacol. Sin 2013;34(9):1229-1236.

This article is protected by copyright. All rights reserved. 
38. McGehee RE, Ronis MJJ, Cowherd RM, Ingelman-Sundberg M, Badger TM. Characterization of cytochrome P450 2E1 induction in a rat hepatoma FGC-4 cell model by ethanol. Biochem. Pharmacol 1994;48(9):1823-1833.

39. Zhukov A, Ingelman-Sundberg M. Selective Fast Degradation of Cytochrome P-450 2E1 in Serum-Deprived Hepatoma Cells by a Mechanism Sensitive to Inhibitors of Vesicular Transport. Eur. J. Biochem 1997;247(1):37-43.

40. Yang MX, Cederbaum AI. Characterization of cytochrome P4502E1 turnover in transfected HepG2 cells expressing human CYP2E1. Arch. Biochem. Biophys 1997;341(1):25-33.

41. Sutendra G, Kinnaird A, Dromparis P, Paulin R, Stenson T, Haromy A, Hashimoto K, et al. A Nuclear Pyruvate Dehydrogenase Complex Is Important for the Generation of Acetyl-CoA and Histone Acetylation. Cell 2014;158(1):84-97.

42. Lavie J, De Belvalet H, Sonon S, Dupuy JW, Lalou C, Dumon E, Melser S, et al. Ubiquitin-Dependent Degradation of Mitochondrial Proteins Regulates Energy Metabolism. Cell Reports 2018;23:2852-2863.

43. Bragoszewski P, Wasilewski M, Sakowska P, Gornicka A, Böttinger L, Qiu J, et al. Retro translocation of mitochondrial intermembrane space proteins. PNAS 2015;112(25):7713-7718.

This article is protected by copyright. All rights reserved. 


\section{FIGURE LEGENDS}

\section{Figure 1. MATa1 is localized to the mitochondrial matrix in liver}

A. Western blot analysis of MATal in whole mouse liver showing both cytosolic and mitochondrial fractions. Mitochondrial (COX IV) and cytosolic (Tubulin) specific markers were immunoblotted to demonstrate fraction purity $(n=3)$. B. Subfractionation of mouse liver $(n=3)$ treated with Proteinase K (PK), Digitonin (Dig) and/or TritonX-100 (TTX). For the different mitochondrial compartments VDAC (outer membrane), COX IV (inner membrane), Cytochrome C (intermembrane space and inner membrane), SDHA (matrix). C. Immunogold-EM of WT mouse liver $(n=2)$ mitochondria showing presence of MAT $\alpha 1$ in the mitochondrial matrix (left panel, distinct concentric circles) as compared to the absence of a specific signal in the no Ab negative control (right panel). Black arrows indicate MAT $\alpha 1$. Images are 80,000X magnification.

Figure 2. MATa1 interacts with heatshock proteins and requires HSP70A8 to gain entry into the mitochondria

A. IP of WT mouse liver lysate $(700 \mu \mathrm{g}, \mathrm{n}=3)$ with anti-MAT $\alpha 1$ or anti-HSP70A8 Ab followed by Western blot analysis with HSP70A8 or MAT $\alpha 1$. B. IP of WT mouse liver lysate (700 $\mu \mathrm{g}, \mathrm{n}=3$ ) with anti-MAT $\alpha 1$ or anti-HSP90 $\beta$ Ab followed by Western blot analysis with HSP90 $\beta$ or MAT $\alpha 1$. Blots were re-probed with Ab used for IP. Normal Ig was used as a negative control, $10 \mu \mathrm{g}$ of lysate used in the IP were loaded as input control. C. Western blot analysis of cytosolic and mitochondria fraction of HepG2 cells $(n=3)$ after silencing of HSPA8 (100 nM) for 72 hours. Tubulin was used a cytosolic marker, while COX IV was used as a mitochondrial marker. Actin was used as housekeeping control to normalize protein expression. D. Graph summarizes the densitometric values expressed as \% of scramble control (SC) from three independent experiments, ${ }^{*} P<0.05$ versus SC. E. As in C but after silencing of HSP90AB1 (30 nM). F. As in D.

This article is protected by copyright. All rights reserved. 
Figure 3. Matla KO mice mitochondria have elevated mROS production and reduced membrane potential

A. Membrane potential and mitochondrial ROS (mROS) of isolated wild type (WT), Matla knockout (KO) hepatocytes and Matla KO hepatocytes overexpressing MAT1A (48 hours, $\mathrm{N}=3) .{ }^{*} P<0.05$ versus $\mathrm{WT},{ }^{\dagger} P<0.05$ versus KO. B. Membrane potential and mROS production were measured in WT mouse hepatocytes where MATlA was either overexpressed (48 hours) or knocked down (66 hours, $50 \mathrm{nM})(\mathrm{n}=3) . * P<0.05$ versus empty vector $(\mathrm{EV}),{ }^{\dagger} P<0.05$ versus scramble control (SC). C. Membrane potential and mROS levels in HepG2 cells with MAT1A knock down (66 hours, $25 \mathrm{nM})(\mathrm{n}=3) .{ }^{*} P<0.05$ versus SC. D. Mitochondrial respiration in isolated liver mitochondria from 7 month old female WT and Matla KO mice. Values are represented as mean \pm SEM ( $\mathrm{n}=6$ replicates from three independent experiments). ${ }^{*} P<0.05$ Matla $\mathrm{KO}$ versus WT. E. Oxygen consumption rate (OCR) measured in HepG2 cells with MAT1A knockdown. Oligomycin (Oligo) inhibits ATP synthesis, FCCP uncouples oxidative phosphorylation, rotenone (Rot) inhibits mitochondrial complex I and antimycin A (AA) inhibits complex III in the respiratory chain. OCR profile was normalized to protein level. Basal and ATP-linked OCR, maximal, and reserve respiratory capacity were calculated. Values are represented as mean \pm SEM $(n=16$ replicates from four independent experiments). ${ }^{*} P<0.05$ versus SiMAT1A.

\section{Figure 4. MATa1 and CYP2E1 directly interact}

In vitro pull-down assay using $\mathbf{A}$. immobilized recombinant MAT $\alpha 1$ with recombinant CYP2E1 or B. immobilized recombinant CYP2E1 with recombinant MAT $\alpha 1$ and IB for MATa1 and CYP2E1 (n=3). C. IP of total mouse liver lysate $(700 \mu \mathrm{g}, \mathrm{n}=3)$ with antiCYP2E1 or anti-MAT $\alpha 1$ Ab followed by Immunoblot (IB) analysis for MAT $\alpha 1$ or CYP2E1. D. IP of isolated WT mouse liver mitochondria $(300 \mu \mathrm{g}, \mathrm{n}=3)$ with anti-CYP2E1 or antiMAT $\alpha 1 \mathrm{Ab}$ followed by IB analysis for MAT $\alpha 1$ or CYP2E1. Normal Ig was used as a negative control and $10 \mu \mathrm{g}$ of lysate was used as input control. Blots were re-probed with Ab used for IP.

This article is protected by copyright. All rights reserved. 
Figure 5. CYP2E1 expression is negatively regulated by MATa1 and MAT1A knockdown-mediated mitochondrial dysfunction is dependent on CYP2E1

A. Relative mRNA expression of 3-month old male Matla and Cyp2e1 in WT and Matla KO liver. Results represent a total of at least three independent experiments done in duplicate. ${ }^{*} P<0.05$ versus WT. B. Protein expression of CYP2E1 in both WT and Matla KO mouse livers, ${ }^{*} P<0.05$ versus WT $(n=3)$. C. Expression of CYP2E1 in isolated mouse liver mitochondria in both WT and Matla KO. ${ }^{*} P<0.05$ versus WT $(n=5)$. Succinate dehydrogenase (SDHA) and COXIV were used as mitochondrial specific markers, and tubulin was used as a cytosolic marker to demonstrate purity. SDHA was used as a housekeeping control to normalize protein expression. D. Mitochondrial CYP2E1 protein quantification of WT and Matla KO mouse liver mitochondria. Peptide to protein rollup was done using MSSTATS. Error bars represent standard deviation from biological replicates. ${ }^{*} P<0.05$ versus WT. E. Western blot analysis of CYP2E1 and MAT $\alpha 1$ in WT mouse hepatocytes, when either Mat1a (66 hours, $50 \mathrm{nM}$ ) and/or Cyp2e1 (66 hours, $30 \mathrm{nM}$ ) were silenced ( $n=3$ ). GAPDH was used as housekeeping control to normalize protein expression. ${ }^{*} P<0.05$ versus double scramble control $(\mathrm{SC}+\mathrm{SC})$. F. Membrane potential and mitochondrial ROS (mROS) levels from experiment described in E. ${ }^{*} P<0.05$ versus double scramble control $(\mathrm{SC}+\mathrm{SC})(n=3)$.

Figure 6. CYP2E1 is a methylated protein and level of me-CYP2E1 is reduced in Mat1a KO liver mitochondria

A. IP of 5-month old male mouse liver mitochondria lysate from WT or Mat1a KO mice $(\mathrm{n}=3)$ with anti-mono/di methyl arginine $\mathrm{Ab}$ followed by immunoblot (IB) analysis for CYP2E1. Amount of methylated CYP2E1 was determined first by normalizing to Ig for each IP and then comparing that value to total levels of CYP2E1 that had been normalized to SDHA. ${ }^{*} P<0.05$ versus WT. B. Intensity of the extracted precursor isotopic envelope (M, $\mathrm{M}+1, \mathrm{M}+2$ ) of a representative CYP2E1 peptide in WT (left panel) and Matla KO (right panel). C. CYP2E1 protein quantification from a mitochondrial methyl-immunoprecipitation using WT and Mat1a KO 5-month old male mouse liver mitochondria. Peptide to protein 
rollup was done using MSSTATS. Error bars represent standard error from biological replicates $(n=4)$. D. Western blot analysis of HepG2 cells that were over-expressed with wither WT CYP2E1, R100N CYP2E1, R379N CYP2E1, or R100N/R379N CYP2E1 mutants for 48 hours in the presence or absence of MAT1A (48 hours) (n=3). GAPDH was used as a housekeeping control to normalize protein expression, ${ }^{*} P<0.05$ versus specific CYP2E1+EV control.

Figure 7. Overexpression of MAT1A enhanced CYP2E1 degradation via the proteasomal pathway

A. Western blot analysis of HepG2 cells that were co-overexpressed with CYP2E1 and MATIA for 48 hours before CHX addition for 5 hours in serum free media $(n=3)$. GAPDH was used as housekeeping control to normalize protein expression. ${ }^{*} P<0.05$ versus CYP2E1+empty vector (EV) control B. Graph shows the $t_{1 / 2}$ for CYP2E1 as compared to EV at time 0 . All densitometric values were normalized to GADPH and expressed as \% of CYP2E1+EV at time 0, average of 3 independent experiments are shown. Regression analysis using the best fit line was done to calculate the half-lives of CYP2E1 with or without MAT1A over-expression. ${ }^{*} P<0.05$ versus CYP2E1+ EV. C. Western blot analysis of HepG2 cells that were over-expressed with or without MAT1A in addition to CYP2E1 for 42 hours before the addition of MG132 for 6 hours $(n=3)$. GAPDH was used as housekeeping control to normalize protein expression. ${ }^{*} P<0.05$ versus CYP2E1 control. D. Western blot analysis of MATal using whole mouse liver showing isolated microsomal fraction. Endoplasmic recticulum marker (Calnexin) and cytosolic marker (Tubulin) were immunoblotted to demonstrate fraction purity $(n=3)$. E. IP of isolated WT mouse liver microsome (300 $\mu \mathrm{g}, \mathrm{n}=3$ ) with anti-CYP2E1 Ab followed by Western blot analysis for MAT $\alpha 1$ or F. IP with anti-MAT $\alpha 1$ Ab followed by Western blot analysis for CYP2E1. Normal Ig was used as a negative control and $10 \mu \mathrm{g}$ of lystate was used as input control. Blots were re-probed with Ab used for IP.

This article is protected by copyright. All rights reserved. 
Figure 8. MAT 1 interacts with CYP2E1 in human liver and methylated CYP2E1 is reduced in human ALD

A. Western blot analysis of MAT $\alpha 1$ in whole human liver $(n=3)$ showing both cytosolic and mitochondrial fractions. Mitochondrial (SDHA) and cytosolic (Tubulin) specific markers were immunoblotted to demonstrate fraction purity. B. IP of total human liver lysate $(700 \mu \mathrm{g}$, $n=3$ ) with anti-MAT $\alpha 1$ or anti-CYP2E1 Ab followed by IB analysis for either CYP2E1 or MAT $\alpha 1$. Blots were re-probed with Ab used for IP. Normal Ig was used as a negative control and $10 \mu \mathrm{g}$ of lysate used as input control. C. Control $(n=4)$ and ALD $(n=5)$ total liver lysate (lys) IB for MAT $\alpha 1$ and densitometric values were normalized to GADPH and expressed as $\%$ over control. D. IP with anti-mono/di methyl arginine Ab followed by IB analysis for CYP2E1. Amount of methylated CYP2E1 was determined first by normalizing to Ig for each IP and then compared to total levels of CYP2E1 that had been normalized to actin. ${ }^{*} P<0.05$ versus control. E. IP of 3.5-month old female mouse liver lysate from pair-fed or NIAAA mice ( $\mathrm{n}=5$ and 6 respectively). IP of total mouse liver lystate $(700 \mu \mathrm{g})$ with anti-mono/di methyl arginine Ab followed by IB analysis for CYP2E1. Amount of methylated CYP2E1 was determined first by normalizing to Ig for each IP and then compared to total levels of CYP2E1 that had been normalized to GAPDH. ${ }^{*} P<0.05$ versus control.

This article is protected by copyright. All rights reserved. 


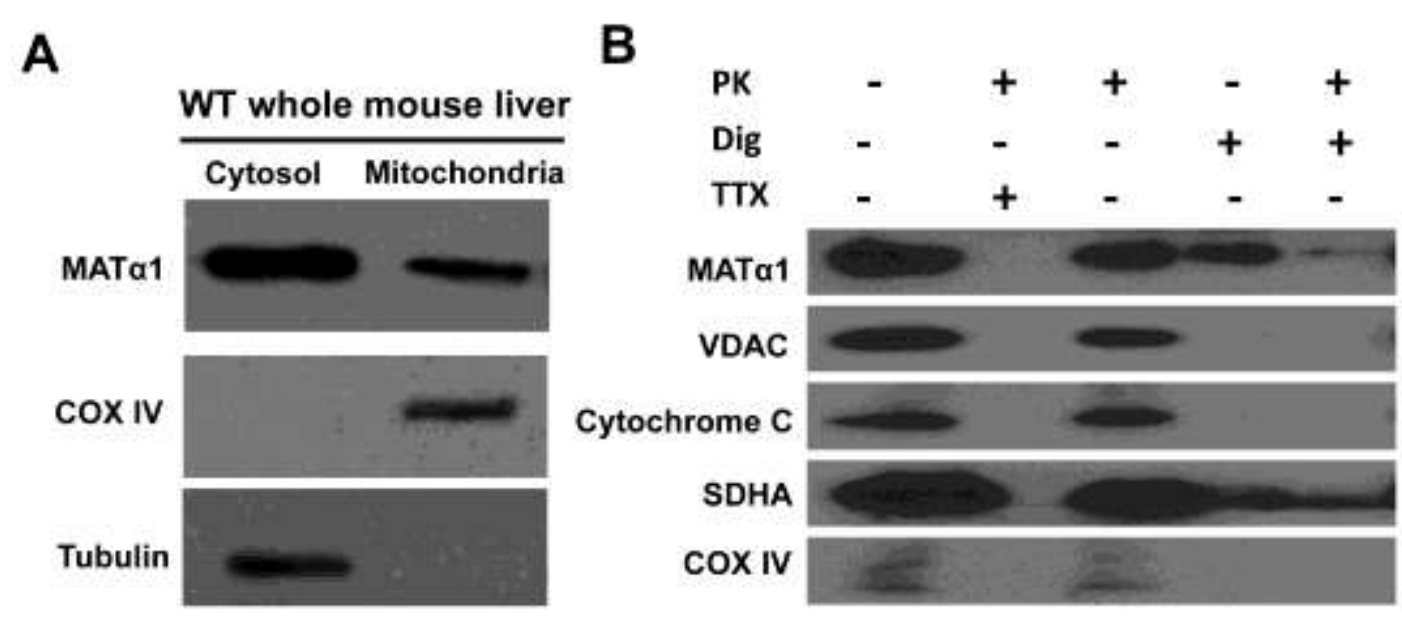

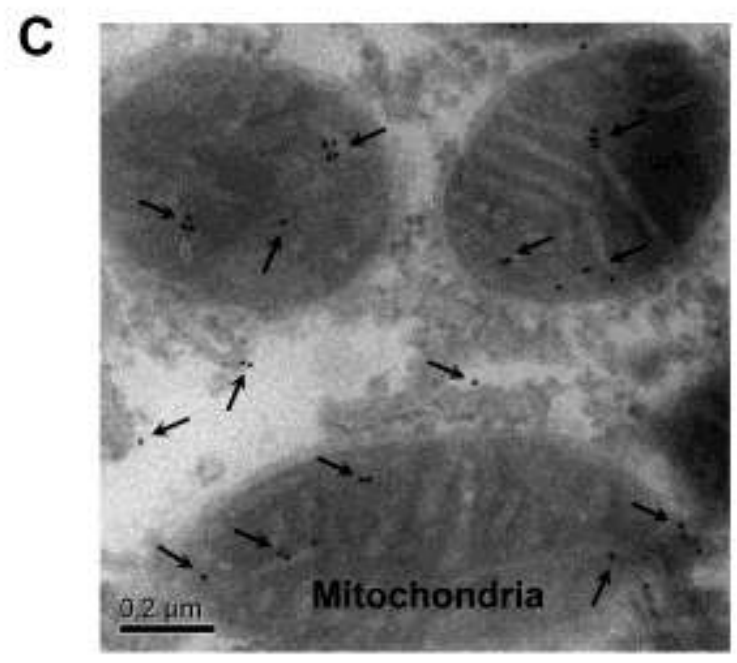

MATal antibody

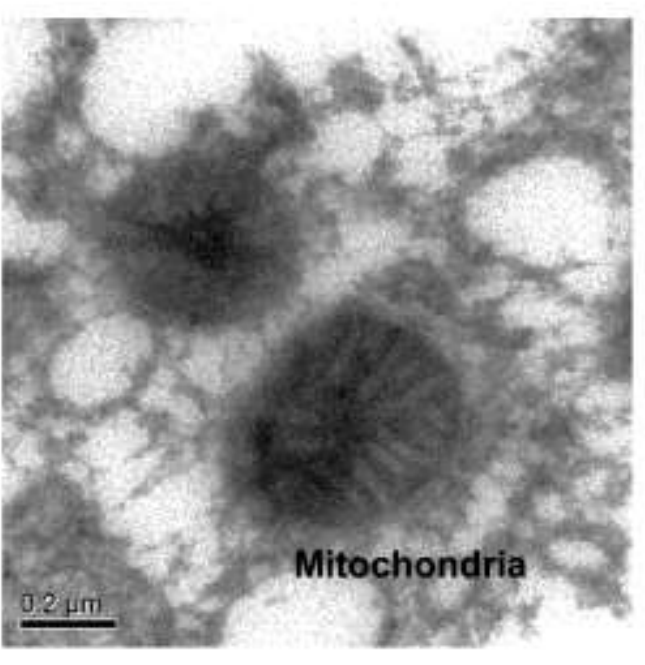

No antibody control

Figure 1

This article is protected by copyright. All rights reserved. 
A Mouse liver

IB Lysate IgG IP:HSP70A8
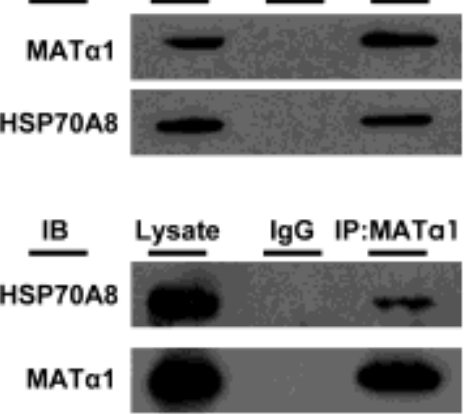

C

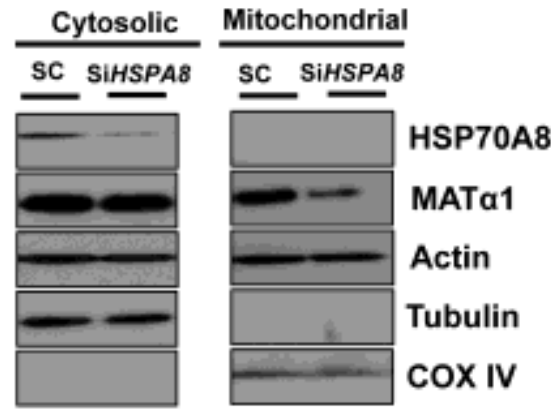

E

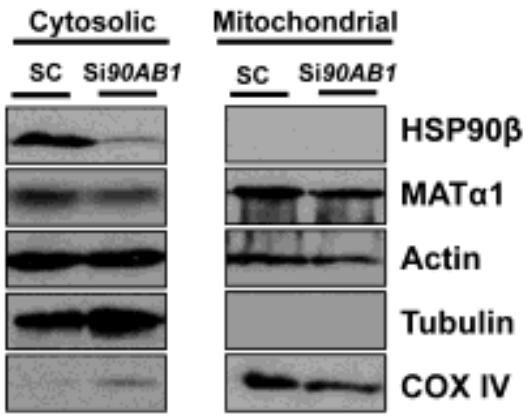

B
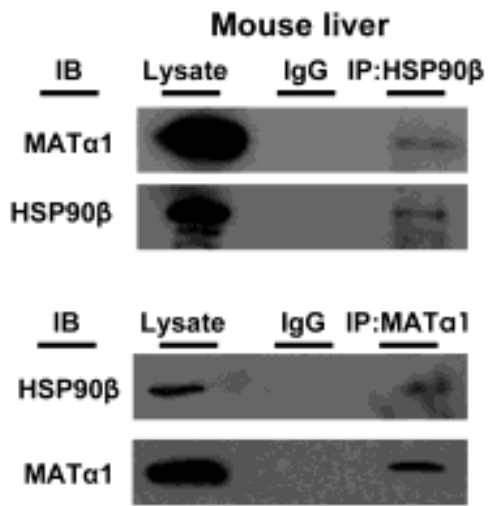

D

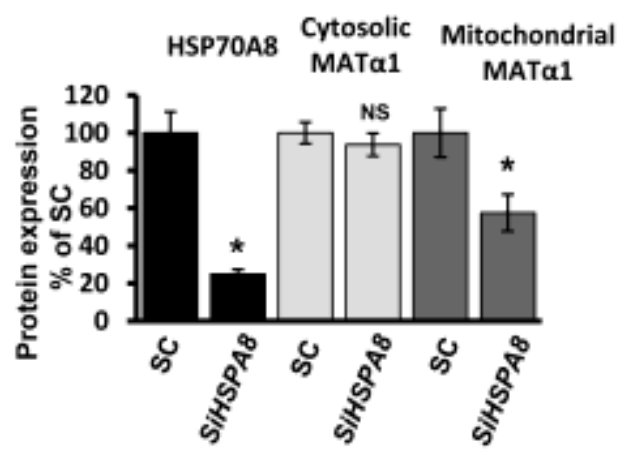

$\mathbf{F}$

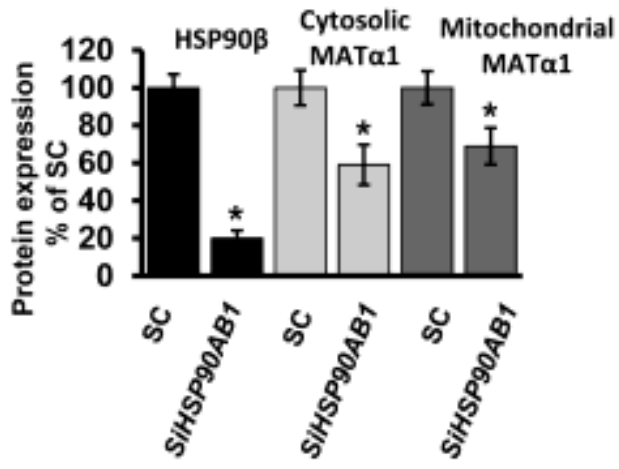

Figure 2

This article is protected by copyright. All rights reserved. 
A
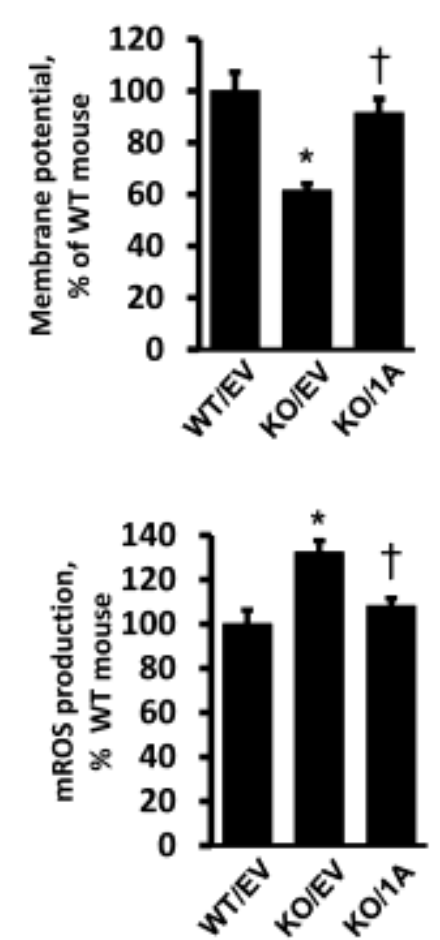

D

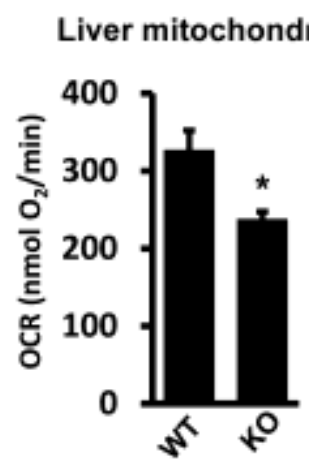

B Mouse hepatocytes
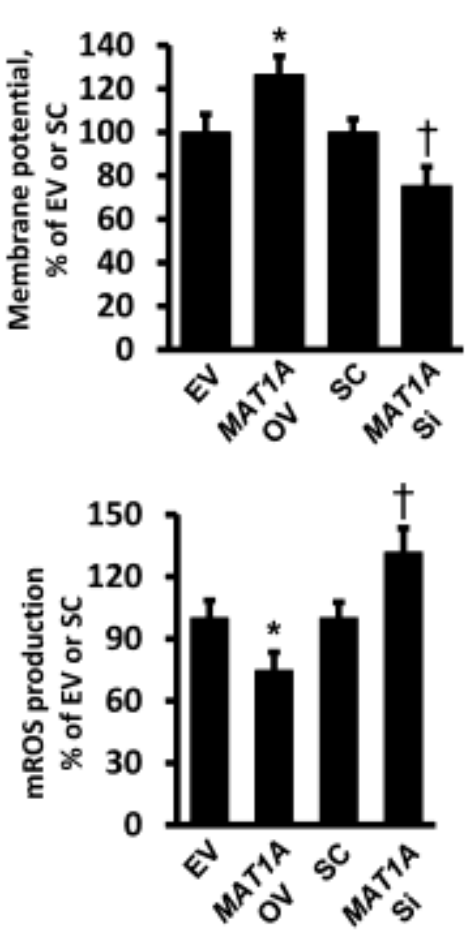

E
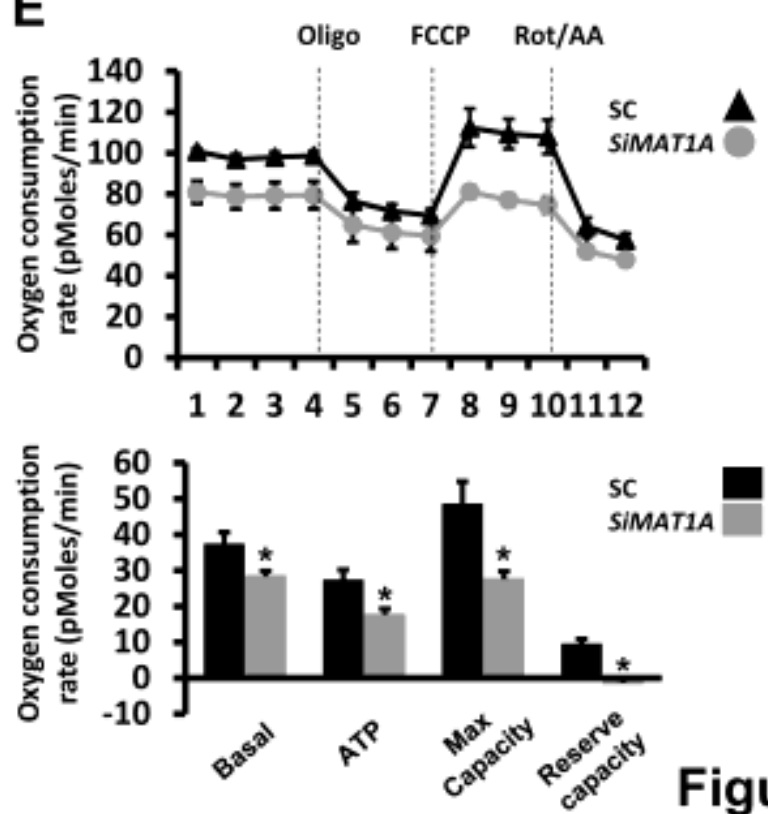

Figure 3 

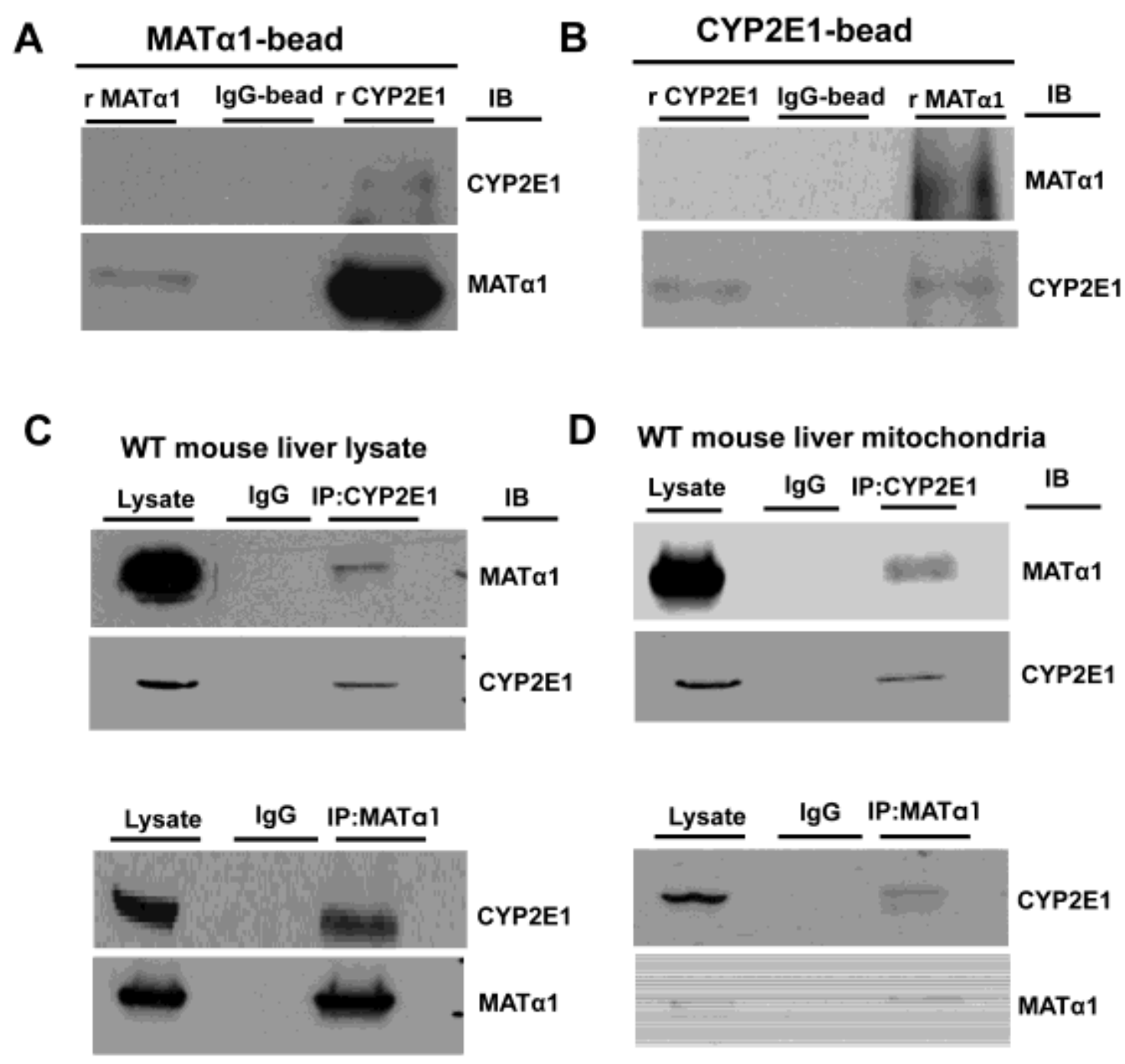

Figure 4

This article is protected by copyright. All rights reserved. 
A

B
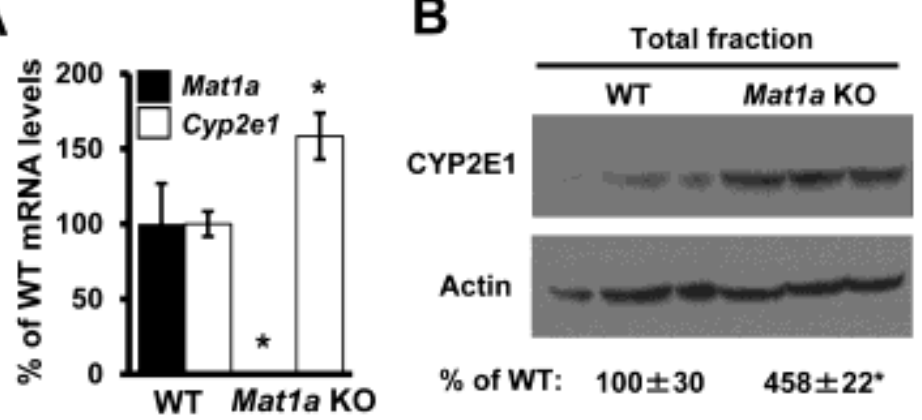

C Mitochondrial fraction

$\%$ of WT: $\quad 100 \pm 30 \quad 458 \pm 22^{*}$

CYP2E1

WT Mat1a KO

SDHA
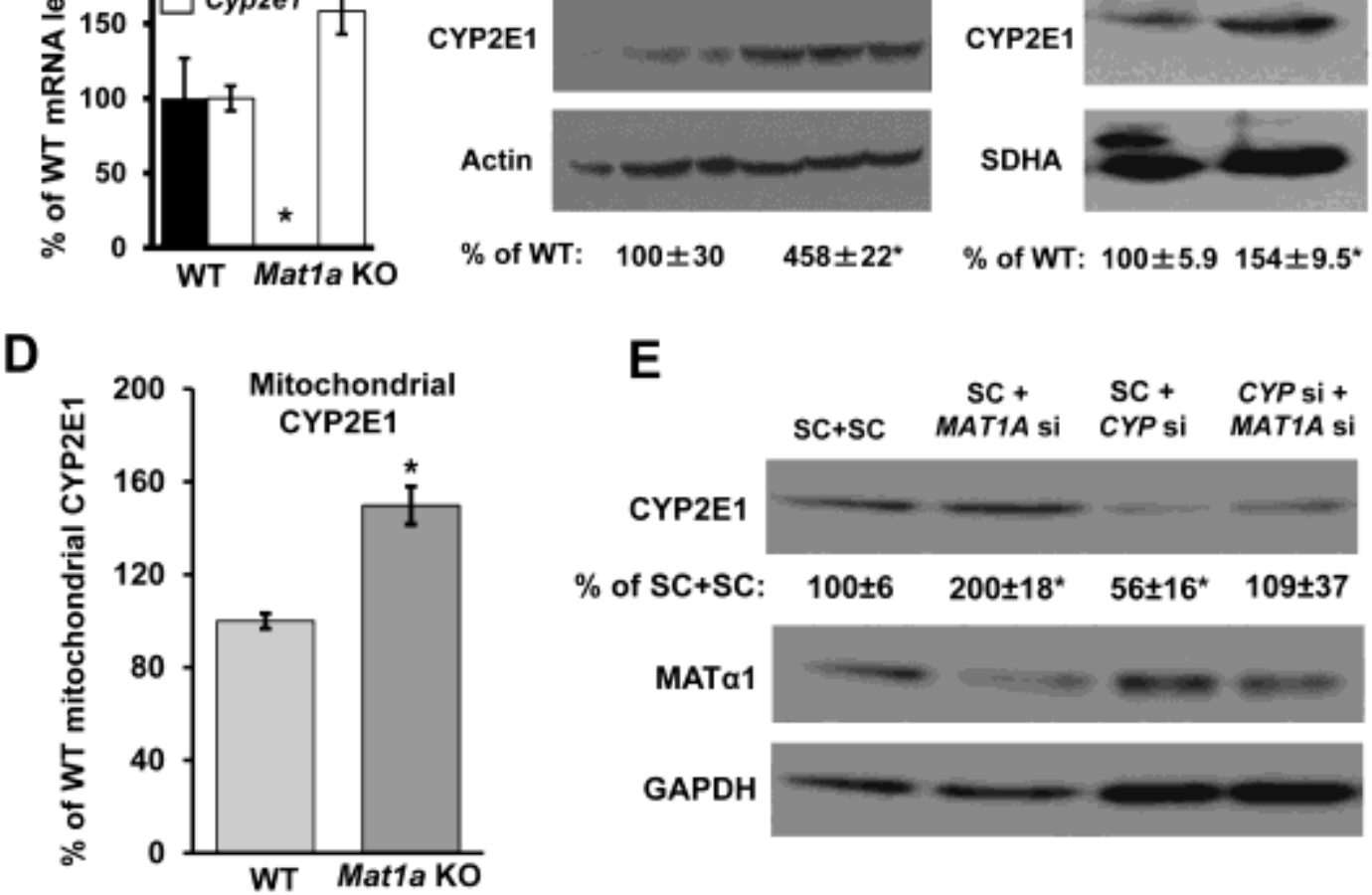

$\mathbf{E}$

$$
\begin{array}{cccc} 
& \mathrm{SC}+ & \mathrm{SC}+ & \text { CYP si }+ \\
\mathrm{sC}+\mathrm{SC} & \text { MAT1A si } & \text { CYP si } & \text { MAT1A si }
\end{array}
$$

CYP2E1

$\%$ of SC+SC: $100 \pm 6 \quad 200 \pm 18^{*} \quad 56 \pm 16^{\star} \quad 109 \pm 37$

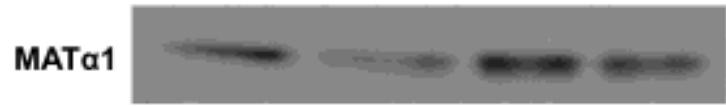

GAPDH

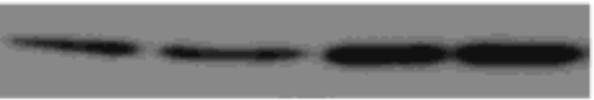

$\mathbf{F}$
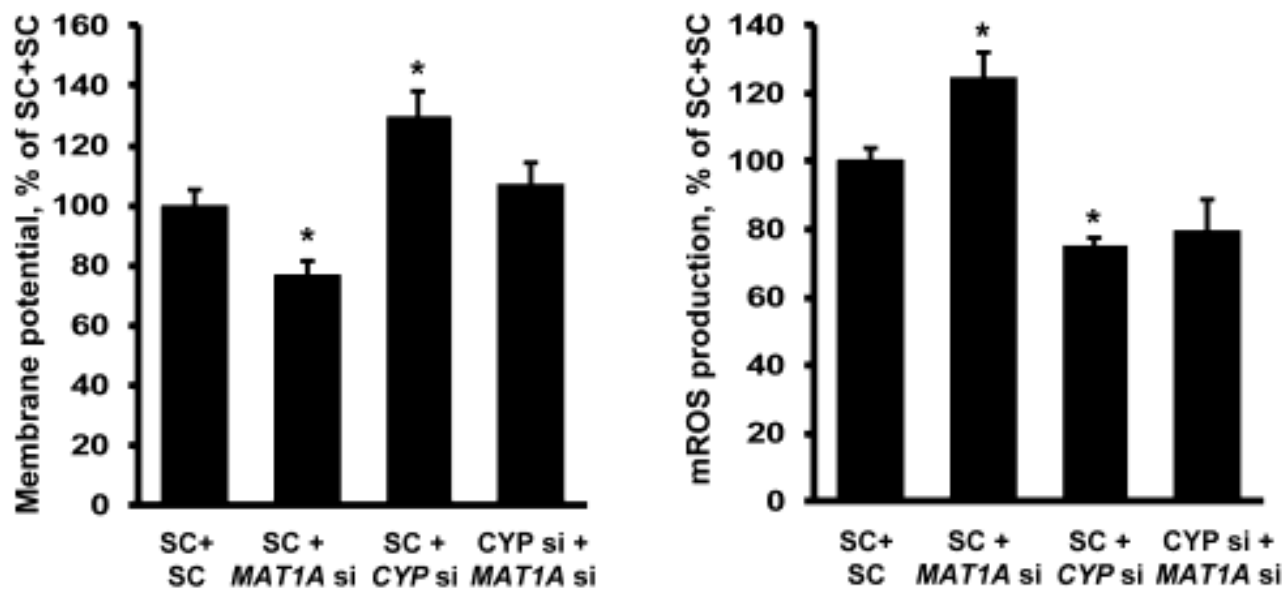

Figure 5

This article is protected by copyright. All rights reserved. 
A

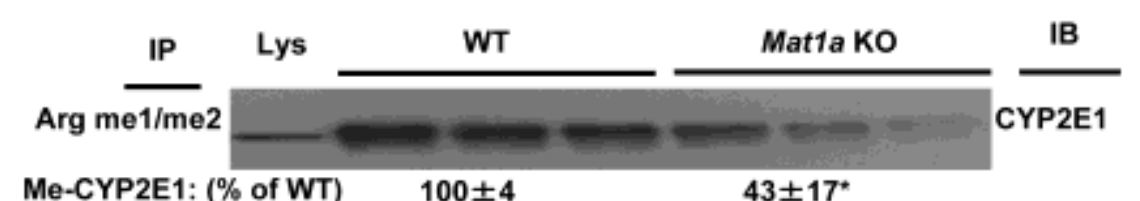

Me-CYP2E1: (\% of WT) $\quad 100 \pm 4 \quad 43 \pm 17^{*}$

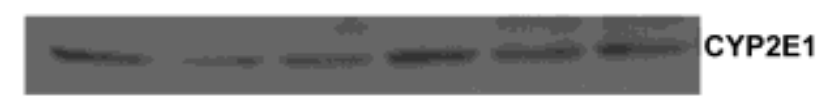

CYP2E1: (\% of WT) $100 \pm 9.5 \quad 128 \pm 3.6^{*}$

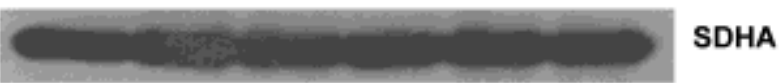

Ratio of Me/total CYP2E1:

(\% over WT)

$100 \pm 5$

$30 \pm 8^{*}$

B

CYP2E1 ${ }^{360}$ FINLVPSNLPHEATR ${ }^{374}$

C Mat1a WT

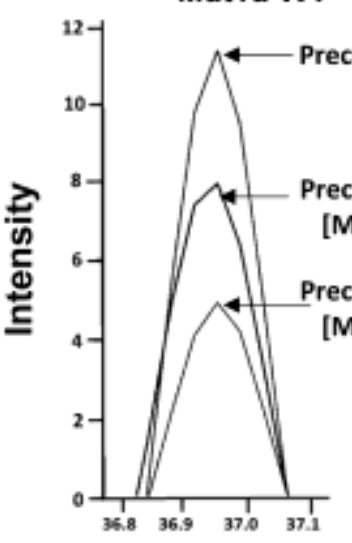

D

D
Mat1a KO

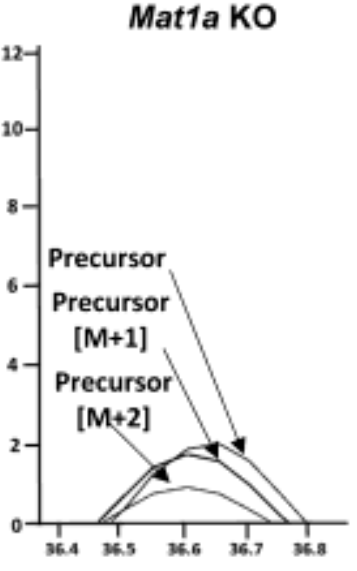

Retention Time (mins)

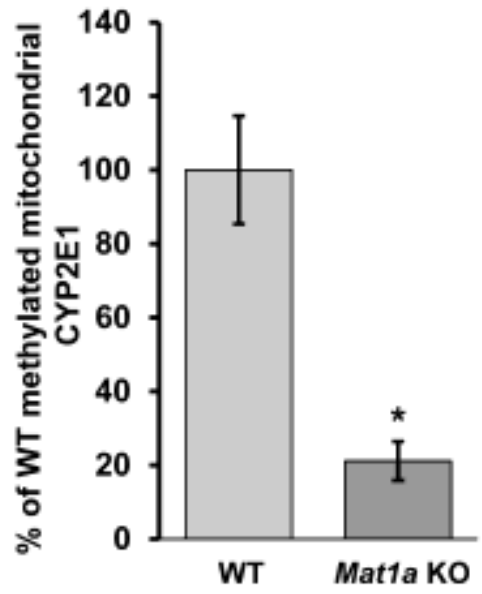

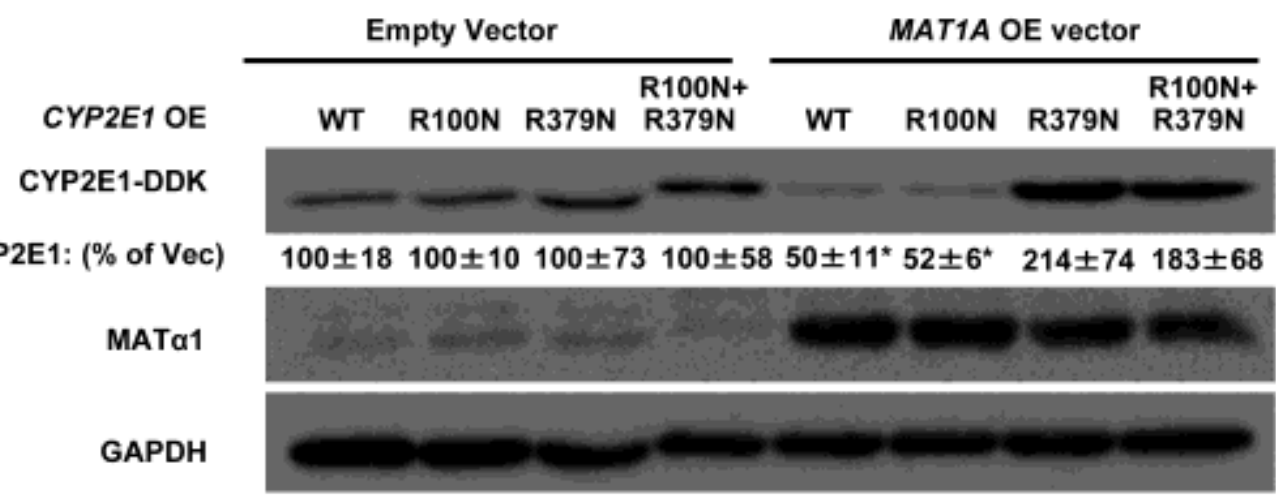

Figure 6

This article is protected by copyright. All rights reserved. 
A

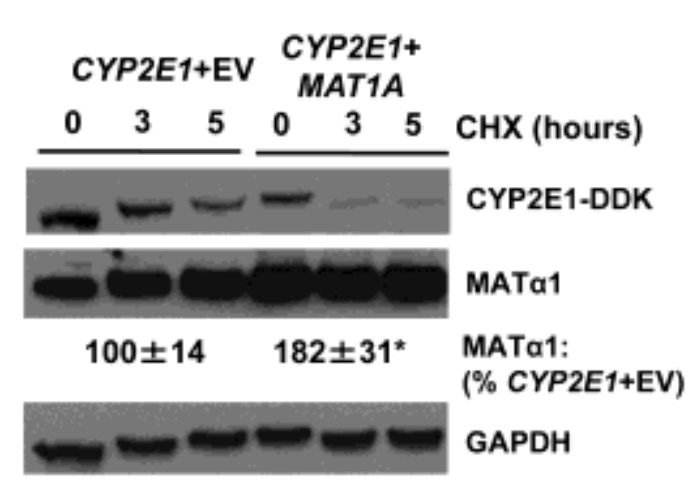

C

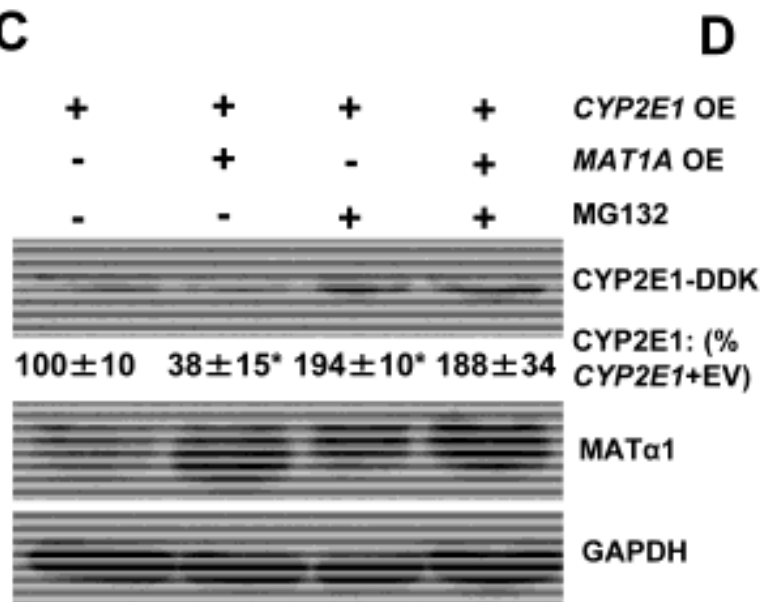

E

WT mouse liver microsome

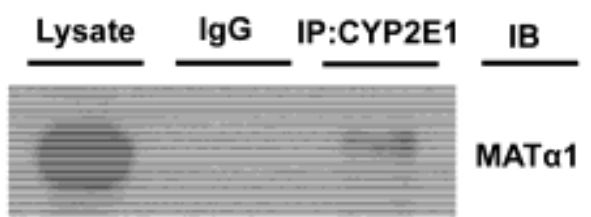

CYP2E1
D

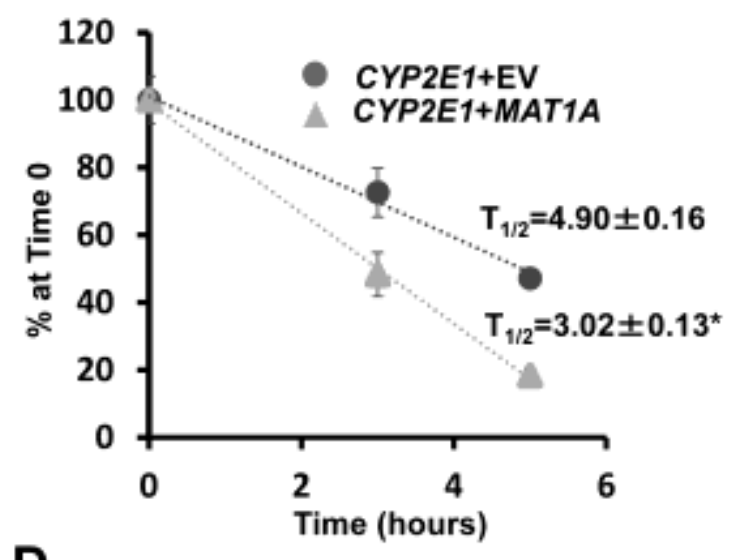

B

WT mouse liver microsome
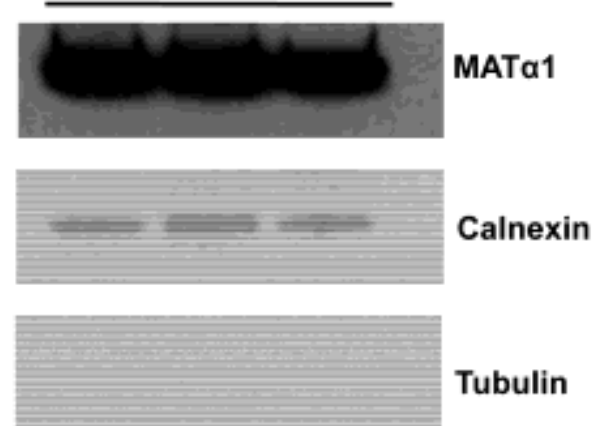

Tubulin

$\mathbf{F}$

WT mouse liver microsome

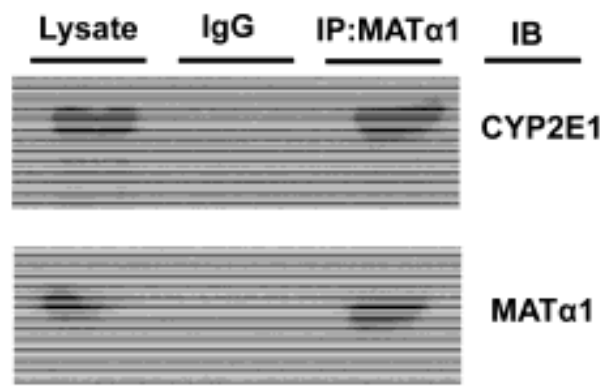

Figure 7

This article is protected by copyright. All rights reserved. 
A

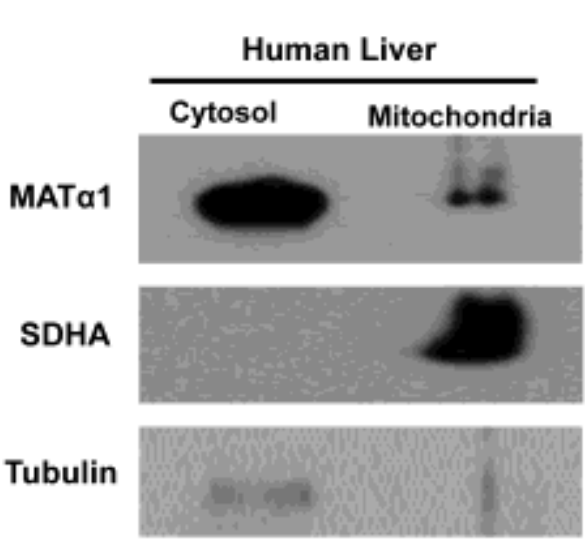

C

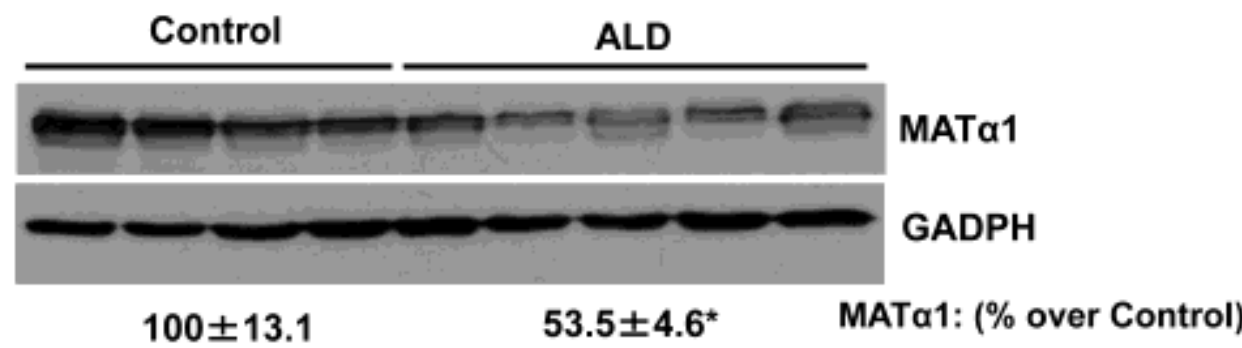

D

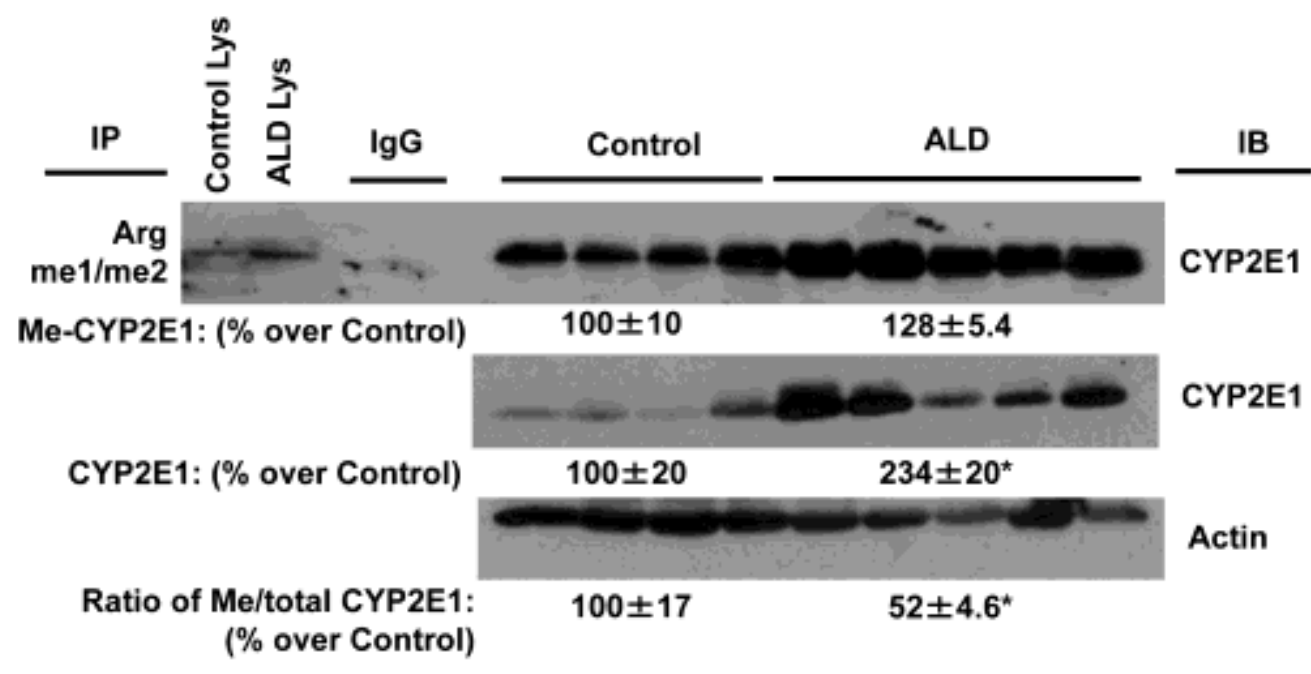

Figure 8

This article is protected by copyright. All rights reserved. 